ITEP Preprint No.: ITEP-TH-34/11

\title{
Random ballistic growth and diffusion in symmetric spaces
}

\author{
A. Gorsky ${ }^{1}$, S. Nechaev ${ }^{2}$, R. Santachiara ${ }^{2,3}$, G. Schehr ${ }^{4}$ \\ ${ }^{1}$ ITEP, B. Cheryomushkinskaya 25, Moscow, Russia \\ ${ }^{2}$ LPTMS, Université Paris Sud, 91405 Orsay Cedex, France \\ ${ }^{3}$ J.-V. Poncelet Laboratory, Independent University of Moscow, \\ 11 B. Vlasievsky per., 119002 Moscow, Russia \\ ${ }^{4}$ LPT, CNRS - Université Paris Sud, 91405 Orsay Cedex, France
}

Sequential ballistic deposition (BD) with next-nearest-neighbor (NNN) interactions in a $N$-column box is viewed a time-ordered product of $N \times N$-matrices consisting of a single $s l_{2}$-block which has a random position along the diagonal. We relate the uniform BD growth with the diffusion in the symmetric space $H_{N}=S L(N, R) / S O(N)$. In particular, the distribution of the maximal height of a growing heap is connected with the distribution of the maximal distance for the diffusion process in $H_{N}$. The coordinates of $H_{N}$ are interpreted as the coordinates of particles of the one-dimensional Toda chain. The group-theoretic structure of the system and links to some random matrix models are also discussed. 
2 A. Gorsky, S.K. Nechaev, R. Santachiara, G. Schehr

\section{CONTENTS}

I. Introduction

II. Ballistic deposition and products of random matrices

4

A. Ballistic growth and directed polymers in random environment

4

B. Ballistic deposition and random walk on local groups

6

C. "Soft deposition"

III. Diffusion in symmetric spaces

10

A. Sequential deposition in two columns and a diffusion in $H=S L(2, R) / S O(2)$

B. Sequential deposition in three columns and diffusion in $H=S L(3, R) / S O(3)$

C. Sequential deposition in $N$ columns and one-dimensional Toda chain

15

D. Toda chain and scaling limit of hyperbolic Calogero-type systems

16

IV. Two views on Lax representation for periodic Toda chain

18

V. Conclusion

21

A. The connection between the columnar heights and coordinates in the symmetric space

B. Minimal action principle for Toda system and rational solutions of Painlevé II

References

\section{INTRODUCTION}

During recent years much effort has been devoted to theoretical analysis of properties of surfaces obtained by aggregation of particles. Several models describing various properties of clusters grown by different deposition processes deserved special attention. Among them we can mention the models of surfaces grown by Molecular Beam Epitaxy (MBE) (see, for example, Ref. [1]), Polynuclear Growth (PNG) [2 [5] and by Ballistic Deposition (BD) [611]. All these models belong to the Kardar-Parisi-Zhang (KPZ) universality class [12]. Our attention is focused on ballistic deposition. In the simplest setting, one can assume that elementary units ("particles") follow ballistic trajectories with random initial positions and specified direction in space and adhere sequentially to a growing aggregate. Despite the extremely transparent geometric formulation, the problem of stochastic growth in the ballistic deposition process remains one of the most challenging subjects in statistical mechanics.

The definition of a standard one-dimensional ballistic deposition model with nextnearest-neighboring (NNN) interactions (for more details, see Refs. [13 15]) is as follows. Consider a box divided in $N$ columns (of unit width each) indexed by an integer $i$ 
$(i=1,2, \ldots, N)$. The free conditions are assumed for left and right boundaries. At the initial time moment, $(t=0)$, the system is empty. Then, at each tick of the clock, $t=1,2, \ldots, T$, we deposit an elementary cell ("particle") of unit height and width in a randomly chosen column, $i$. Suppose that the distribution of these nucleations on the set of columns is uniform. Define the height, $h_{i}(t)$, in the column $i$ at time moment $t$. Assume now, that the cells in the nearest-neighboring columns interact in such a way that they can only touch each other by corners, but never by their vertical sides. This implies that after having deposited a particle to the column $i$, the height of this column is modified according to the following rule:

$$
h_{i}(t+1)=\max \left[h_{i-1}(t), h_{i}(t), h_{i+1}(t)\right]+1 .
$$

If at the time moment $t$ nothing is added to the column $i$, its height remains unchanged: $h_{i}(t+1)=h_{i}(t)$. A set of deposited particles forms a heap (a pile) as it is depicted in Fig. 2 b.

The available theoretical analysis of stochastic deposition focuses mainly on the properties of the enveloping surface $h_{i}(T)$. Recall the well-known scaling relation characterizing the surface of a growing aggregate:

$$
[\operatorname{Var} h(T)]^{1 / 2}=\frac{1}{N^{1 / 2}}\left[\sum_{i=1}^{N}\left\langle h_{i}^{2}(T)\right\rangle-\left\langle h_{i}(T)\right\rangle^{2}\right]^{1 / 2}=N^{1 / 2} g\left(\tau / N^{3 / 2}\right),
$$

where the brackets denote an average over different realizations of the noise, the variable $\tau=T / N$ is the averaged number of particles per one column, and the function $g(u)$ is the rescaled variable $u=\tau / N^{3 / 2}$ has the following asymptotic behavior: $g(u) \sim u^{1 / 3}$ for $u \ll 1$ and $g(u) \sim$ const for $u \gg 1$. Such a behavior is typical for many non-stationary growth problems. The exponent 1/3 has been found already in [12] for the original Kardar-ParisiZhang (KPZ) model and then observed in a variety of other growth models. In [16, 17] it was realized that, for flat initial conditions, the distribution of a rescaled PNG surface height, $\tau^{-1 / 3}(h(i, \tau)-2 \tau)$, converges as $\tau \rightarrow \infty$ to the Tracy-Widom (TW) distribution [18], describing the edge states of random matrices belonging to the Gaussian Orthogonal Ensemble (GOE). Note that in the droplet geometry the statistics of growing surface is instead described by the edge states of the Gaussian Unitary Ensemble (GUE) [17]. Height fluctuations in the KPZ universality class were measured in experiments, both in planar [19 21] and in curved geometry in the electroconvection of nematic liquid crystals [20, 21] and a good quantitative agreement with TW distributions was found.

The typical analytic analysis of a growth process described by the equation (1) consists of a few heuristic steps which are usually beyond a precise control. First of all, one sends the lattice spacing and time step to zero (with proper rescaling of all relevant variables), thus arriving at a continuous nonlinear partial differential equation of the type

$$
\partial_{t} h(x, t)=\nu \partial_{x}^{2} h(x, t)+g\left|\partial_{x} h(x, t)\right|+\eta(x, t),
$$

where $\nu$ and $g$ are some constants and $\eta(x, t)$ is a Gaussian white noise, which replaces the telegraph noise in the initial equation (1). The next step is to replace the nonanalytic nonlinearity $\left|\partial_{x} h(x, t)\right|$ by the analytic one $\left(\partial_{x} h(x, t)\right)^{2}$. This replacement is based on numerical evidence [22] and solid mathematical estimates [23] which show that the universality class of KPZ equation is insensitive to the value of $\alpha(1 \leq \alpha \leq 2)$ for the nonlinearity of the type $\left|\partial_{x} h(x, t)\right|^{\alpha}$. 
Another point of view on ballistic deposition process has been developed recently in [24], where it has been demonstrated that the discrete stochastic equation describing the BD process in (11) can be naturally represented in terms of a "dynamic programming" language associated with the so-called Bellman equation [25]. This "dynamic programming" point of view allowed for a systematic translation of evolution of growing BD clusters in the NNN BD model into the language of maximizers and shocks in discrete equations of the Burgers or Hamilton-Jacobi type. This approach allowed to determine the exponents characterizing: i) the decay of the number density of clusters, ii) the wandering of the inter-cluster interface, and iii) the tail of the cluster mass distribution.

In the present paper we consider the ballistic deposition process from a completely different point of view, linking it to the diffusion in some symmetric space. The goal of our work is two-fold:

1. On one hand, we conjecture that the uniform NNN ballistic growth in a scaling limit can be interpreted as multiplication of random matrices of type (11), which, in turn is linked to the diffusion in the symmetric space $H_{N}=S L(N, R) / S O(N)$. In particular, the distribution of the maximal height of a growing heap is connected with the distribution of the maximal distance for the diffusion process in $H_{N}$;

2. On the other hand, we demonstrate that the coordinates of $H_{N}$ can be interpreted as the coordinates of particles of the one-dimensional Toda chain, which itself can be regarded as Inozemtsev limit of Calogero-type systems describing the transmission in random wires.

The paper is structured as follows. In the Section II we remind the concept of "local groups" and demonstrate that the BD process can be described in terms of matrix multiplications; in the Section [II we describe the NNN BD process as diffusion in an appropriate symmetric space, derive the connection with the one-dimensional Toda system and show that BD can be described by the Inosemtsev limit of hyperbolic Calogero-type systems; in the Section [V] we interpret some group-theoretic facts concerning Toda chain in terms of ballistic deposition; the Conclusion summarizes our results. The two Appendices outline: a) the connection between heights and eigenvalues, and b) the link between 1D Toda system and rational solutions of Painlevé II.

\section{BALLISTIC DEPOSITION AND PRODUCTS OF RANDOM MATRICES}

\section{A. Ballistic growth and directed polymers in random environment}

Consider the set of matrices $\left\{g_{1}, \ldots, g_{N}\right\}$, where the matrix $g_{i}$ has the following form:

$$
g_{i}=\left(\begin{array}{lllllll}
1 & & & & & & \\
& \ddots & & & & & \\
& & & & & & \\
& & 1 & 0 & 0 & & \\
& & u & u & u & & \\
& & 0 & 0 & 1 & & \\
& & & & & \ddots & \\
& & & & & & 1
\end{array}\right) \leftarrow \text { row } i .
$$


The deposition event in the column $i$ means the application of the generator $g_{i}$. Rising a heap by sequential addition of particles with NNN interactions is translated into a sequential multiplication of matrices of type (44). Thus, the whole heap is represented by the timeordered product

$$
V(T, u)=: \prod_{t=1}^{T} g_{i_{t}}:
$$

Each element of the matrix $V(T, u)$ is a polynomial of the variable $u$. Take a vector $\mathbf{a}(t=$ $0)=\left(a_{1}, \ldots, a_{N}\right)$ where $a_{i}(i=1, \ldots, N)$ are strictly positive values. The set of local heights $\mathbf{h}(T)=\left(h_{1}(T), \ldots, h_{N}(T)\right)$ at time $T$ after deposition event can be extracted as follows:

$$
\mathbf{h}(T)=\lim _{u \rightarrow \infty} \frac{\ln [V(T, u) \mathbf{a}(t=0)]}{\ln u} .
$$

The definition of heights in (44)-(6) is consistent with the updating rules (11) for the NNNballistic deposition process. Namely, write the recursion for the updating dynamics of the vector $\mathbf{a}(t)$ :

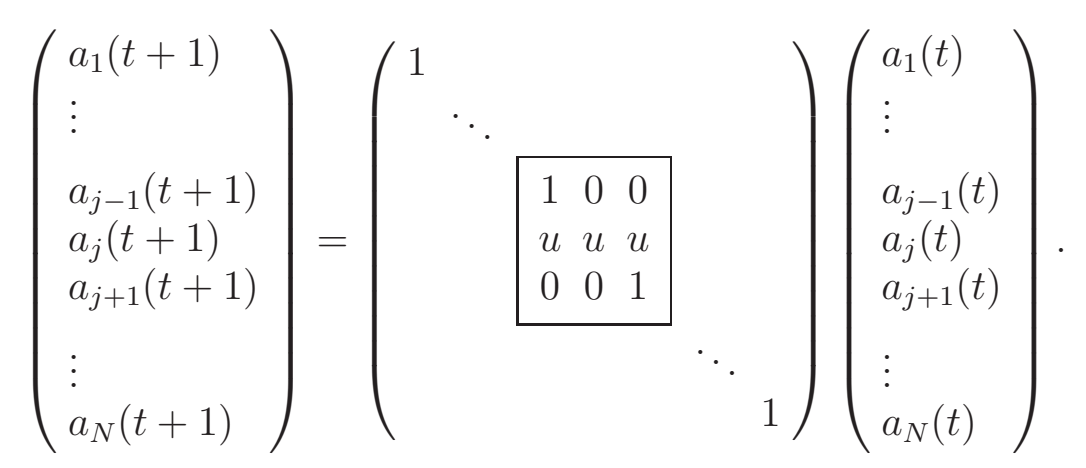

If $i_{t}=i$ (i.e. we drop a particle in the column $i$ ar time $t$ ), then

$$
a_{i}(t+1)=u a_{i-1}(t)+u a_{i}(t)+u a_{i+1}(t) .
$$

Otherwise $a_{i}(t+1)=a_{i}(t)$. Substituting the ansatz $a_{i}(t)=e^{\beta h_{i}(t)}$ into (8) , we get for deposition event in the column $i$ :

$$
h_{i}(t+1)=\frac{1}{\beta} \ln \left[e^{\beta h_{i-1}(t)}+e^{\beta h_{i}(t)}+e^{\beta h_{i+1}(t)}\right]+1 .
$$

For all other columns $j \neq i$ the height remains unchanged: $h_{j}(t+1)=h_{j}(t)(j=1, \ldots, N)$. The initial configuration by definition is $\mathbf{h}(T)=(0, \ldots, 0)$. Taking in (9) the limit $\beta \rightarrow \infty$, we recover (10). Note, that (9) resembles the ultra-discretization of stochastic growth equations [26].

The quantity $a_{i}(t)$ can be interpreted as a finite temperature partition function of a directed polymer in a random environment, $\beta=\log u$ being essentially the inverse temperature. To demonstrate this consider the random lattice depicted in the Fig. 1, The rules of the deposition process mean that at each "time" $t$ a single site (an open dot) is chosen: an energy $-\epsilon$ is assigned to this site and it is connected to three neighboring ancestors at time $t-1$. To the other sites (black dots) an energy 0 is assigned and they are connected (vertically) to a single site. On this random lattice we then consider a configuration of a directed polymer, shown by a bold line. 
6 A. Gorsky, S.K. Nechaev, R. Santachiara, G. Schehr

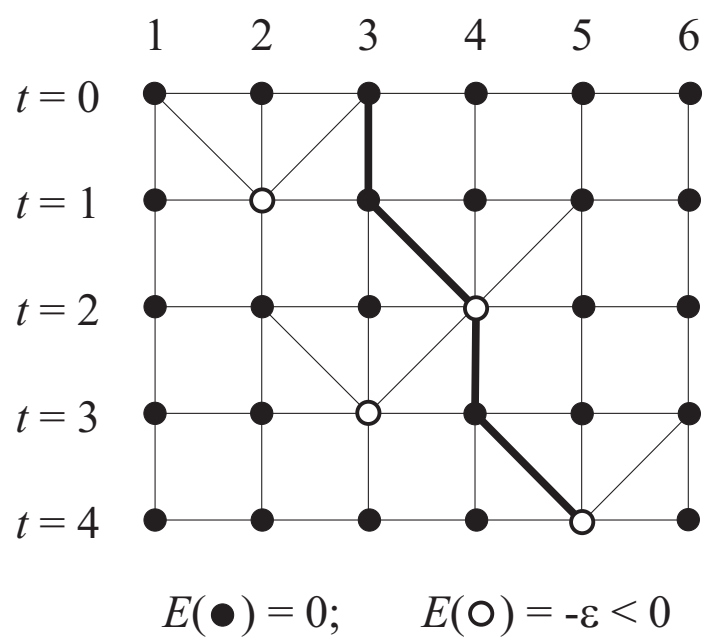

Figure 1. Directed polymer model in a disordered environment. Open dots designate the randomly placed sites with energy $-\epsilon$.

The energy assigned to a polymer configuration is the sum of site energies through which the directed polymer is passing. If we denote by $e^{\beta \epsilon}=u$, then the quantity $a_{i}(t)$ evolving according to (7) gives exactly the partition function of a polymer ending in site $i$ at time t. By this correspondence a precise connection between the $\mathrm{BD}$ a directed polymer in a random medium is established (see, for instance, [27] and references therein).

\section{B. Ballistic deposition and random walk on local groups}

We have seen that the ballistic deposition process has a natural description in terms of multiplication of random matrices with special "local" structure. The established connection is a particular example of a uniform Markov process on a "local group" [28].

Definition 1. Local group (semi-group) $L_{N}\left(L_{N}^{+}\right)$with $N$ generators $g_{1}, \ldots, g_{N}$ is a group (semi-group) with the commutation relations

$$
g_{k} g_{m}=g_{m} g_{k}, \quad \forall|k-m| \geq 2,
$$

and might have additional relations $R$ inside the elementary blocks $g_{k}, g_{k \pm 1}(k=1, \ldots N)$. If the relations $R$ are the same for all $k$, then $L$ is called a local stationary group [28].

Plenty of important groups, semi-groups and algebras are of type of local groups: the Coxeter groups, Hecke algebra, braid groups, Temperley-Lieb algebra, etc. A locally free group $F$ with $M$ generators, being in some sense the "simplest" nontrivial example of a local group, can be considered as a universal object in the manifold of all local groups with a given number of generators.

Definition 2. Locally free group (semi-group) $F_{N}\left(F_{N}^{+}\right)$with $N$ generators $g_{1}, \ldots, g_{N}$ is a group (semi-group), determined only by the relation (10). Each pair of neighboring generators $g_{k}, g_{k \pm 1}$ produces a free subgroup (sub-semigroup) of $F_{N}\left(F_{N}^{+}\right)$[29].

Let us define the numbered heap as a heap whose cells are enumerated by sequential "ticks of time", $t$. Deposition of a particle in a column $i$ means the application of a generator $g_{i}$. 
There is a bijection between numbered heaps of $T$ sequentially deposited cells and words of length $T$ in $F_{N}^{+}$such that a given numbered heap is uniquely represented by a word in $F_{N}^{+}$ (and vice versa) [29]. The key point of this bijection is the special way of representing words in the locally free semigroup (group). Namely, all words are written in the so-called "normal order form" implying that the generators with smaller indices are pushed as left as possible when it is allowed by commutation relations (10). For example, the word associated with subsequent deposition of all 13 particles in Fig. 2 is: $W=g_{3} g_{6} g_{1} g_{4} g_{1} g_{2} g_{5} g_{3} g_{1} g_{5} g_{3} g_{6} g_{2}$. The same word $W$ written in the normal order form $W=g_{1} g_{1} g_{3} g_{2} g_{1} g_{4} g_{3} g_{3} g_{2} g_{6} g_{5} g_{5} g_{6}$, is in bijection with the numbered heap (Fig. 2b) obtained by "contraction" of the sequential deposition (Fig. 2a). The statistical properties of locally free semigroups and groups are investigated in details in [29]. Thus, the uniform growth of a random heap on a set of $N$ columns can be considered as a uniform Markov chain on a semigroup $F_{N}^{+}$with $N$ generators.
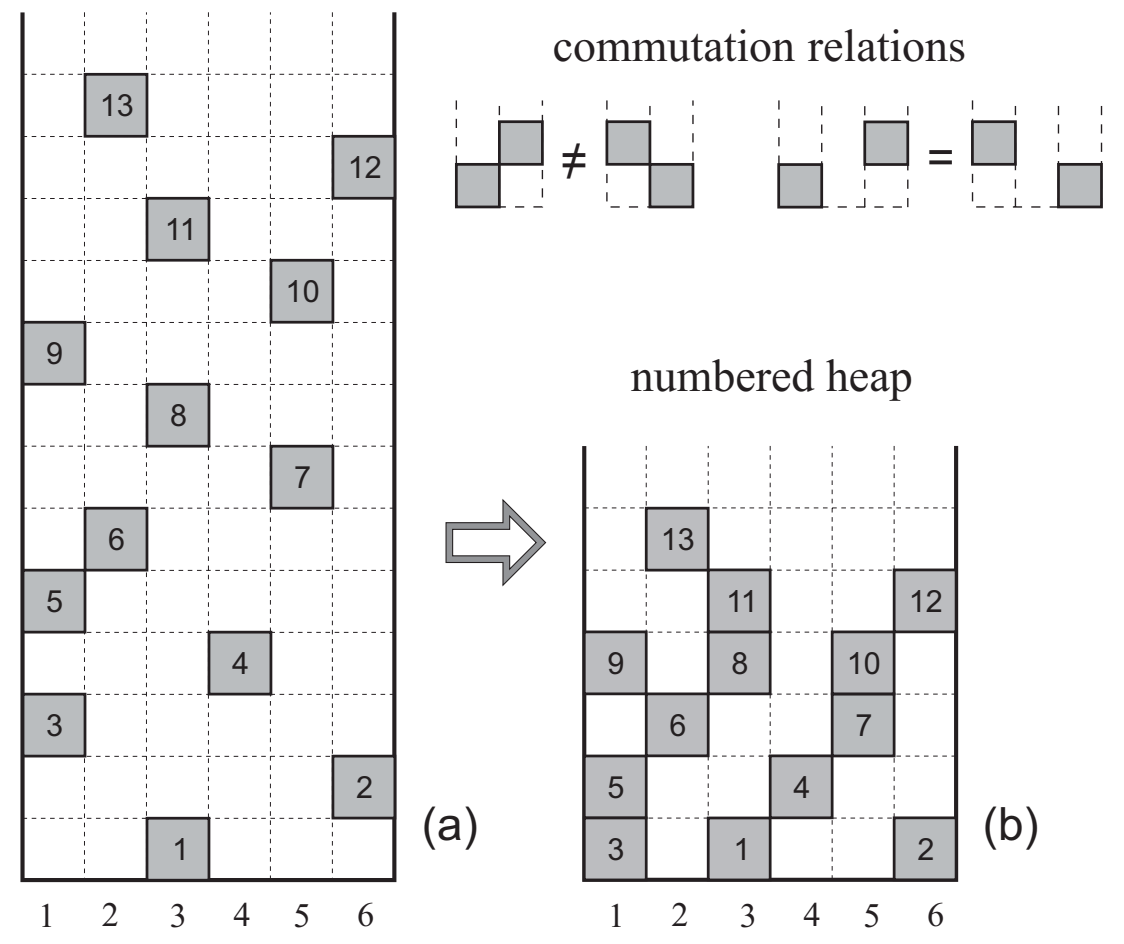

(a)

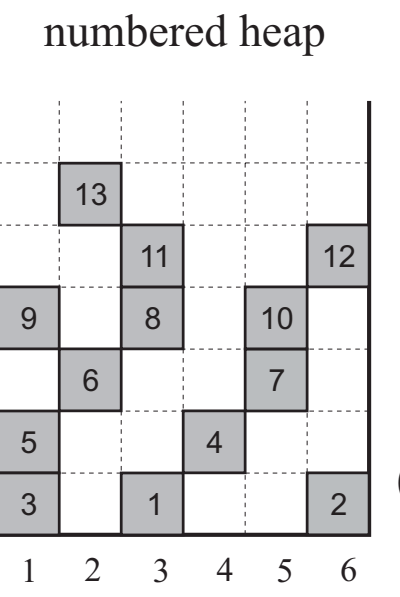

(b)

Figure 2. (a) The sequential ballistic deposition; (b) the heap obtained by contraction procedure using commutation relations of $F_{N}^{+}(N=6)$.

It should be noted that the concept, equivalent to the locally free semi-group $F_{N}^{+}$, appeared earlier [30] in the investigations of combinatorial properties of substitutions of sequences and so called "partially commutative monoids" [31]. Especially productive is the geometrical interpretation of monoids in the form of a "heap", offered by X.G. Viennot [30]. It is worth mentioning that the concept of a monoid-heap appears in connection with various aspects of directed growth, parallel computing and complexity algorithms [32].

Definition 3. Take a locally free semi-group $F_{N}^{+}$with fixed finite number of generators $\left\{g_{1}, \ldots, g_{N}\right\}$. Consider the uniform distribution on the set $\left\{g_{1}, \ldots, g_{N}\right\}$. Construct the (lefthanded) Markov chain on $F_{N}^{+}: \mathbf{a}(t=0)=\left\{a_{1}, \ldots, a_{N}\right\}, \mathbf{a}(t)=g_{i_{t}} \mathbf{a}(t-1)$, where $i_{t}=$ $\{1, \ldots, N\}$ is uniformly distributed on the set of indices $\{1, \ldots, N\}$.

Analogously with locally free semi-group, we can define the Markov chain on a locally free 
group. The geometric interpretation of random walk on locally free group is very straightforward. Consider two types of particles: black and white, and identify the generators $g_{i}$ and $g_{i}^{-1}$ respectively with deposition of black and white particle in the column $i(i=1, \ldots, N)$. The elementary depositions are depicted in Fig. 3, where (a) corresponds to the deposition $g_{1} g_{2} g_{2}^{-1} \equiv g_{1}\left(\right.$ since $\left.g_{i} g_{i}^{-1}=1\right)$, and the configuration shown in (b) cannot be reduced.

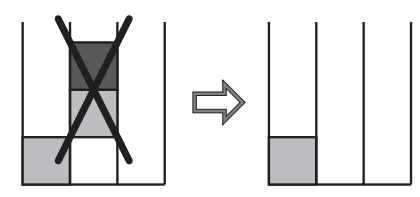

(a)

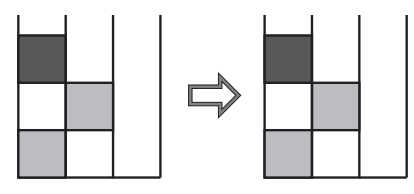

(b)

Figure 3. Deposition rules for colored heap (annihilation is allowed).

\section{C. "Soft deposition"}

Deposition process viewed as a random walk on a locally free semigroup (or group) can be considered from the point of view of diffusion in symmetric spaces. Let us demonstrate this on two simple examples: sequential deposition in two and three columns.

Deposition in two columns corresponds to a Markov chain on a locally free semigroup $F_{2}^{+}$with two generators, $g_{1}, g_{2}$ can be represented by a Cayley tree shown in Fig. 4 a, since for $N=2$ one has $F_{2}^{+} \equiv \Gamma_{2}^{+}$, where $\Gamma_{2}^{+}$is a free semigroup with two generators (see, for example, [33] for definitions). Deposition in three columns can be treated similarly as a Markov chain on the Cayley graph associated with locally free semigroup $F_{3}^{+}:\left\{g_{1}, g_{2}, g_{3}\right\}$ and the commutation relation $g_{1} g_{3}=g_{3} g_{1}$ - see Fig. 4b. Certainly, the same correspondence can be established between the ballistic deposition with annihilation (as shown in Fig. 3) and random walk on the full locally free group $F_{N}$.

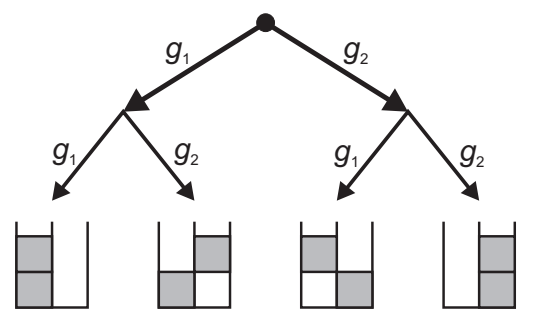

(a)

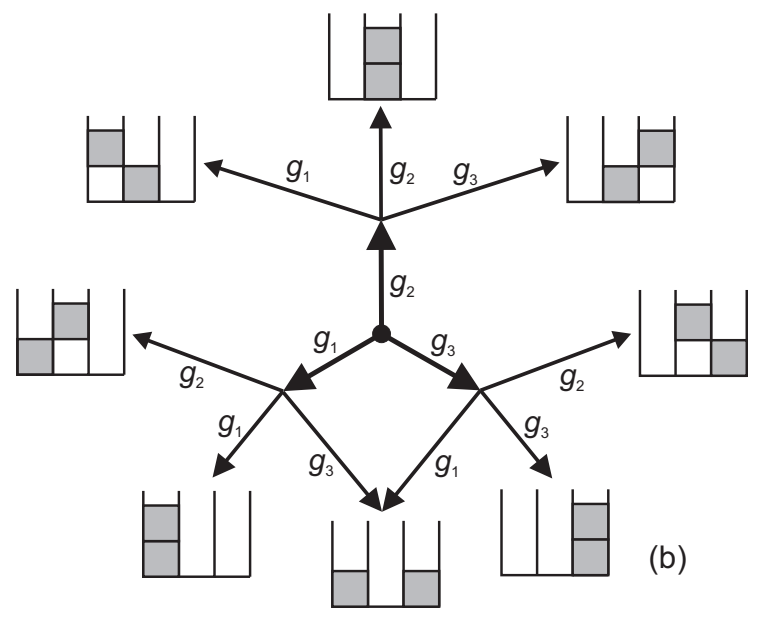

Figure 4. Ballistic deposition in two (a) and three (b) columns labelled by states of Cayley graphs of corresponding locally free semigroups.

We shall also consider a random walk on the full group $F_{N}$, i.e. the uniform growth in a two-color heap in which direct deposition of white particle on top of black one (and vice versa) leads to their mutual annihilation. 
The key problem connecting the $N$-column deposition and diffusion in symmetric space is the following. For the multi-column deposition we need to find a metric space, $H_{N}$ in which the locally free group $F_{N}$ with $N$ generators acts as a group of isometries, i.e. in which the Cayley graph $\mathcal{C}_{N}$ of $F_{N}$ can be isometrically embedded.

It is well known that any regular Cayley tree, $\mathcal{C}$, as an exponentially growing structure, cannot be isometrically embedded in an Euclidean plane. The embedding of a Cayley tree $\mathcal{C}$ into the metric space is called "isometric" if $\mathcal{C}$ covers that space, preserving all angles and distances. For example, the rectangular lattice isometrically covers the Euclidean plane $E=\{x, y\}$ with the flat metric $d s^{2}=d x^{2}+d y^{2}$. In the same way, the Cayley tree $\mathcal{C}$ isometrically covers the surface of constant negative curvature (the Lobachevsky plane) $\mathcal{H}$. One of possible representations of $\mathcal{H}$ is the Poincaré model, the upper half-plane $\operatorname{Im} z>0$ of the complex plane $z=x+i y$ endowed with the metric $d s^{2}=\frac{d x^{2}+d y^{2}}{y^{2}}$ of constant negative curvature. Thus, the locally free group $F_{2} \equiv \Gamma_{2}$ is the group of isometries of Lobachevsky plane. Since the Lobachevsky plane is the coset space $H_{2}=S L(2, R) / S O(2)$, we can consider the two-column ballistic deposition (with possible cancellation) as a random walk in $\mathrm{H}_{2}$.

Similarly, the locally free group $F_{3}$ generated by $g_{1}, g_{2}, g_{3}$ with the commutation relation $g_{1} g_{3}=g_{3} g_{1}$ acts as a group of isometries of the Lobachevsky space with the metric $d s^{2}=\frac{d x^{2}+d y^{2}+d z^{2}}{y^{2}}$. Thus, the deposition in three columns has an important difference with respect to the two-columnar growth. Namely, the steps along the coordinates $x$ and $z$ in Lobachevsky space commute which is straightforwardly translated into the commutation relation $g_{1} g_{3}=g_{3} g_{1}$ in the group $F_{3}$. Hence, the three-columnar growth corresponds to the symmetric random walk in Lobachevsky space $H_{3}=S L(3, R) / S O(3)$. The consideration of the corresponding diffusion equations in curved geometry is the subject of the next Section.

To exploit the power of algebraic methods, we suggest the following smoothed description of $N$-columnar ballistic growth.

Definition 4. We define the "soft" deposition generator, $\tilde{g}_{i}$, as follows:

$$
\tilde{g}_{i}(t)=\left(\begin{array}{ccccc}
1 & & & & \\
& \ddots & & & \\
& & & & \\
& & U_{i}^{(t)} & & \\
& & & \ddots & \\
& & & & 1
\end{array}\right) ; \quad U_{i}^{(t)}=\left(\begin{array}{cc}
a_{i}^{(t)} & b_{i}^{(t)} \\
c_{i}^{(t)} & d_{i}^{(t)}
\end{array}\right) ; \quad a_{i}^{(t)} d_{i}^{(t)}-b_{i}^{(t)} c_{i}^{(t)}=1
$$

where the block $U_{i}^{(t)}$ is an $S L(2, R)$ matrix. For definiteness, suppose that the entries $a_{i}^{(t)}, b_{i}^{(t)}, c_{i}^{(t)}, d_{i}^{(t)}$ are random variables (up to the condition $\operatorname{det} U_{i}^{(t)}=1$ ) distributed in a compact support with some measure $P(u)$.

Note that this "smoothed" description captures all principal properties of the growing heap which we would like to analyze:

i) $\tilde{g}_{i}\left(t_{1}\right) \tilde{g}_{j}\left(t_{2}\right)=\tilde{g}_{j}\left(t_{1}\right) \tilde{g}_{i}\left(t_{2}\right)$ for $|i-j| \geq 2$ and $t_{2}>t_{1}$, meaning that sequential deposition in distant (i.e. non-nearest-neighboring) columns commutes;

ii) $\tilde{g}_{i}\left(t_{1}\right) \tilde{g}_{j}\left(t_{2}\right) \neq \tilde{g}_{j}\left(t_{1}\right) \tilde{g}_{i}\left(t_{2}\right)$ for $|i-j|=1$ and $t_{2}>t_{1}$, meaning that sequential deposition in nearest-neighboring columns does not commute. 
The group $\tilde{G}:\left\{\tilde{g}_{1}, \ldots, \tilde{g}_{N}\right\}$ falls under the Definition 1 of local groups. The advantage of the redefinition of deposition generators $g_{i} \rightarrow \tilde{g}_{i}$ [compare (4) and (11)] will appear below. Equipped with these definitions, we formulate the following conjecture:

Conjecture. The Cayley graphs associated to the locally free group generated by $g_{i}$ 's on one hand, and to the local group generated by $\tilde{g}_{i}$ 's on the other hand, can both be embedded isometrically in the same metric space $H_{N}$.

The metric space $H_{N}$ is the symmetric space $H_{N}=S L(N, R) / S O(N)$. One can then use the Lie group structure of this symmetric space to define the Beltrami-Laplace operator which coincides with the Casimir operator $C_{2}$. This would allow us to describe the symmetric Markov chain on the locally free group $F_{N}$ with $N$ generators by the uniform diffusion process in the symmetric space $H_{N}$, where the group $F_{N}$ acts as a group of isometries.

The connection between the columnar heights, $h_{j}(t)$ (defined in (9)), and current coordinates $\mu_{j}(t)$ describing the diffusion process in the symmetric space $H_{N}$, is as follows. Denote by $\tilde{V}_{t}$ the time-ordered product of $t$ generators $\tilde{g}$ in (11). Let $\left\{\lambda_{1}(t), \ldots, \lambda_{N}(t)\right\}$ be the set of eigenvalues of the matrix $\tilde{V}_{t}$; define $\mu_{j}(t)=\ln \lambda_{j}(t)(j=1, \ldots, N)$. Then, one has for $t \gg 1$ :

$$
h_{\max }(t)=\gamma_{N} \mu_{\max }(t)
$$

where $h_{\max }(t)=\max \left\{h_{1}(t), \ldots, h_{N}(t)\right\}, \mu_{\max }(t)=\max \left\{\mu_{1}(t), \ldots, \mu_{N}(t)\right\}$, and $\gamma_{N}$ is some constant which depends only on the matrix size, $N$. Our numerical computations show that $\gamma_{N}$ converges rapidly to its limiting value $\gamma \approx 0.8$ as $N$ increases - see Appendix $\mathrm{A}$. We also describe in Appendix $\mathrm{A}$ the relation (for $N=2$ ) between the number of steps, $n$, from the origin along a Cayley tree embedded into $H_{2}=S L(2, R) / S O(2)$, and a hyperbolic distance $d$ in $\mathrm{H}_{2}$.

\section{DIFFUSION IN SYMMETRIC SPACES}

\section{A. Sequential deposition in two columns and a diffusion in $H=S L(2, R) / S O(2)$}

For $N=2$ the sequential deposition process is viewed as a multiplication of $S L(2, R)-$ random matrices. The equation for the stochastic evolution of the vector $\mathbf{r}(t)=(x(t), y(t))$ is

$$
\left(\begin{array}{l}
x(t+1) \\
y(t+1)
\end{array}\right)=\left(\begin{array}{ll}
a^{(t)} & b^{(t)} \\
c^{(t)} & d^{(t)}
\end{array}\right)\left(\begin{array}{l}
x(t) \\
y(t)
\end{array}\right) .
$$

which can be conveniently rewritten, introducing the Ricatti variable $\rho(t)=x(t) / y(t)$, as:

$$
\rho(t+1)=\frac{a^{(t)}+b^{(t)} \rho(t)}{c^{(t)}+d^{(t)} \rho(t)}
$$

The stochastic equation (13) was the subject of the pioneering work [34] where it has been explicitly shown that the limiting distribution function $W_{T}(\rho)$ for $T \gg 1$ and $a_{i}^{(t)}, b_{i}^{(t)}, c_{i}^{(t)}, d_{i}^{(t)}$ randomly distributed on a compact support with one and the same distribution for all $t=1, \ldots, T$, satisfies the diffusion equation in the Lobachevsky plane (the two-dimensional 
Riemann surface of constant negative curvature). Recall that the Lobachevsky plane coincides with the symmetric space $H_{2}=S L(2, R) / S O(2)$. So, we could immediately write the heat equation in $H_{2}$ for the distribution function $P(\mathbf{x}, t)$, where $t$ is the "time" and $\mathbf{x}$ are the coordinates of a Riemann manifold:

$$
\partial_{t} P(\mathbf{x}, t)=D \Delta_{\mathrm{BL}} P(\mathbf{x}, t) ; \quad P(\mathbf{x}, t=0)=\delta(\mathbf{x})
$$

where

$$
\Delta_{\mathrm{BL}}=\frac{1}{\sqrt{g}} \frac{\partial}{\partial x_{i}}\left(\sqrt{g}\left(g^{-1}\right)_{i k} \frac{\partial}{\partial x_{k}}\right)
$$

is the Beltrami-Laplace operator and $g_{i k}$ is the metric tensor of the manifold; $g=\operatorname{det} g_{i k}$. The function $P(\mathbf{x}, t)$ satisfies the normalization condition $\int \sqrt{g} W(\mathbf{x}, t) d \mathbf{x}=1$.

It seems to be instructive to outline the derivation of (15) using the standard technique of extracting the Lie algebra generators from the appropriate matrix decomposition and writing the corresponding Casimir operator. Let us note that the proof of convergence and existence of limiting distribution associated with the stochastic equation (14) was a subject of the work [35].

The $s l_{2}$ Lie algebra is defined by the relations [36]

$$
\left[X_{1}, X_{2}\right]=X_{3} ; \quad\left[X_{1}, X_{3}\right]=-2 X_{1} ; \quad\left[X_{2}, X_{3}\right]=2 X_{2},
$$

where the generators $X_{1}, X_{2}, X_{3}$ have the $2 \times 2$ matrix representation

$$
X_{1}=\left(\begin{array}{cc}
0 & 1 \\
0 & 0
\end{array}\right) ; \quad X_{2}=\left(\begin{array}{cc}
0 & 0 \\
1 & 0
\end{array}\right) ; \quad X_{3}=\left(\begin{array}{cc}
1 & 0 \\
0 & -1
\end{array}\right) \text {. }
$$

As we shall see later, the geometry of the system presumes the use of Gauss matrix decomposition [36]. So, we represent the $S L(2, R)$-matrix $U$ as:

$$
U \equiv\left(\begin{array}{ll}
a & b \\
c & d
\end{array}\right)=\left(\begin{array}{ll}
1 & 0 \\
y & 1
\end{array}\right)\left(\begin{array}{cc}
e^{ \pm \mu} & 0 \\
0 & e^{\mp \mu}
\end{array}\right)\left(\begin{array}{ll}
1 & x \\
0 & 1
\end{array}\right)
$$

In the differential form (in the right representation) the $s l_{2}$-generators $X_{1}, X_{2}, X_{3}$ read:

$$
X_{1}=\partial_{x} ; \quad X_{2}=e^{\mp 2 \mu} \partial_{y} \pm x \partial_{\mu}-x^{2} \partial_{x} ; \quad X_{3}=-2 x \partial_{x} \pm \partial_{\mu} .
$$

The Casimir $C_{2}$, which coincides with the Beltrami-Laplace operator $\Delta_{\mathrm{BL}}$, can be expressed as follows

$$
C_{2}=\Delta_{\mathrm{BL}}=X_{1} X_{2}+X_{2} X_{1}+\frac{1}{2} X_{3}^{2}=\frac{1}{2} \partial_{\mu}^{2} \pm \partial_{\mu}+2 e^{\mp 2 \mu} \partial_{x} \partial_{y} .
$$

Thus, the diffusion equation for the distribution function $W(\mu, x, y, t)$ has the following form

$$
\partial_{t} W(\mu, x, y, t)=D\left(\frac{1}{2} \partial_{\mu}^{2} \pm \partial_{\mu}+2 e^{\mp 2 \mu} \partial_{x} \partial_{y}\right) W(\mu, x, y, t)
$$

where $D$ is the diffusion coefficient. Let us make a comment concerning two options to choose the sign in equations (19)-(22). It is obvious that in (19) the sign can be chosen arbitrary, however in (20)-(22) the sign should be consistent with correct physical behavior of the system at large times. The simplest way to chose the correct sign is to compare the 
asymptotic behavior of $W$ with the limiting distribution of large number of noncommuting matrices. The classical paper by H. Fürstenberg [37] states, that under some natural conditions typical products of $T$ noncommuting matrices $[S L(2, R)$ belongs to the class under consideration] increase exponentially with $T$. This selects $C_{2}=\frac{1}{2} \partial_{\mu}^{2}-\partial_{\mu}+2 e^{2 \mu} \partial_{x} \partial_{y}$. Seeking now the solution of (22) in the form $W(\mu, x, y, t)=e^{a t+b \mu} Z(\mu, x, y, t)$ and choosing $a=-\frac{D}{2}, b=1$ we arrive at the Liouville-type equation for the function $U(\mu, x, y, t)$ :

$$
\partial_{t} Z(\mu, x, y, t)=\frac{D}{2}\left(\partial_{\mu}^{2}+4 e^{2 \mu} \partial_{x} \partial_{y}\right) Z(\mu, x, y, t)
$$

In what follows we are interested in the $\mu$-dependence ("radial part") of the function $U$ only. In this case the "angular" dependence on $x$ and $y$ can be eliminated by the reduction conditions [38], which imply the replacement $\partial_{x} \partial_{y} \rightarrow \varkappa^{2}=$ const. Thus we arrive at the equation

$$
\partial_{t} Z(\mu, t)=\frac{D}{2}\left(\partial_{\mu}^{2}+4 \varkappa^{2} e^{2 \mu}\right) Z(\mu, t)
$$

subject to the initial condition $Z(\mu, 0)=\delta(\mu)$. The solution of (24) is well known and can be written explicitly, for example, as in [39]

$$
\begin{aligned}
& Z(\mu, t)=\frac{1}{2 \pi} \int_{-\infty}^{\infty} d k \psi_{k}(\mu) \psi_{k}^{*}(0) e^{-k^{2} t} \\
& \psi_{k}(\mu)=2 \sqrt{\frac{2 k}{D \pi} \sinh \frac{2 k \pi}{\sqrt{D}}} K_{2 i k / \sqrt{D}}\left(4 \sqrt{\frac{\varkappa^{2}}{D}} e^{\mu}\right),
\end{aligned}
$$

where $K_{a}(z)$ is the Macdonald function. For generic forms of solution of (24) see [40].

\section{B. Sequential deposition in three columns and diffusion in $H=S L(3, R) / S O(3)$}

As we have seen in Introduction, for $N=3$ the sequential deposition process is not reduced to a random walk in $H_{2}$ and the situation is more involved. If one considers two sequential deposition events, we can encounter with equal probability all configurations schematically depicted below. Namely, besides the former $S L(2, R)$ matrix multiplication

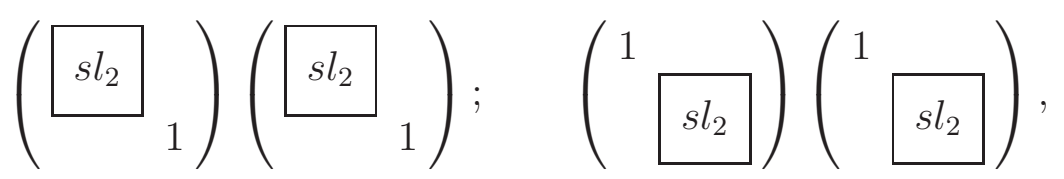

we have also the matrix multiplication of two "shifted" copies $S L(2, R)_{+} \otimes S L(2, R)_{-}$, where the notation $+(-)$ designates upper (lower) $s l_{2}$-block

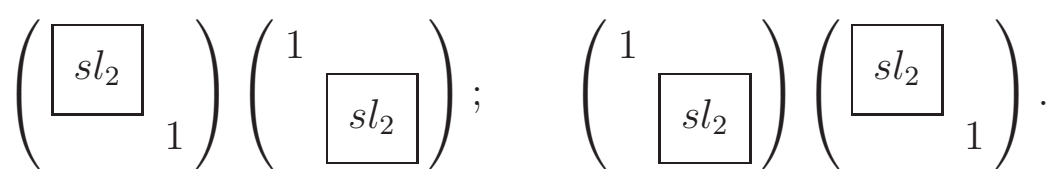

It can be easily demonstrated that the multiplications of type (26) -(27) do generate the whole group $S L(3, R)$. We use the Lie algebra representation of two "shifted" algebras $s l_{2}$ 
and check that all $s l_{3}$ generators can be obtained only from the multiplications of type (26) -(27). Using $s l_{2}$-representation (18) we construct the set of generators of two "shifted" copies of $s l_{2}$ :

$$
\begin{aligned}
& X_{1}=\left(\begin{array}{lll}
0 & 1 & 0 \\
0 & 0 & 0 \\
0 & 0 & 0
\end{array}\right) ; X_{2}=\left(\begin{array}{lll}
0 & 0 & 0 \\
1 & 0 & 0 \\
0 & 0 & 0
\end{array}\right) ; X_{3}=\left(\begin{array}{ccc}
1 & 0 & 0 \\
0 & -1 & 0 \\
0 & 0 & 0
\end{array}\right) ; \\
& Y_{1}=\left(\begin{array}{lll}
0 & 0 & 0 \\
0 & 0 & 1 \\
0 & 0 & 0
\end{array}\right) ; Y_{2}=\left(\begin{array}{lll}
0 & 0 & 0 \\
0 & 0 & 0 \\
0 & 1 & 0
\end{array}\right) ; Y_{3}=\left(\begin{array}{ccc}
0 & 0 & 0 \\
0 & 1 & 0 \\
0 & 0 & -1
\end{array}\right)
\end{aligned}
$$

These generators satisfy the commutation relations of $s l_{3}$ algebra:

$$
\begin{gathered}
{\left[X_{1}, X_{2}\right]=X_{3} ; \quad\left[X_{1}, X_{3}\right]=-2 X_{1} ; \quad\left[X_{2}, X_{3}\right]=2 X_{2} ;} \\
{\left[Y_{1}, Y_{2}\right]=Y_{3} ; \quad\left[Y_{1}, Y_{3}\right]=-2 Y_{1} ; \quad\left[Y_{2}, Y_{3}\right]=2 Y_{2} ;} \\
{\left[X_{1}, Y_{3}\right]=X_{1} ; \quad\left[X_{2}, Y_{3}\right]=-X_{2} ; \quad\left[Y_{1}, X_{3}\right]=Y_{1} ; \quad\left[Y_{2}, X_{3}\right]=-Y_{2}}
\end{gathered}
$$

However the list (28) does not enumerates all generators of $s l_{3}$ : two generators of $s l_{3}, X_{4}$ and $Y_{4}$, where

$$
X_{4}=\left(\begin{array}{ccc}
0 & 0 & 1 \\
0 & 0 & 0 \\
0 & 0 & 0
\end{array}\right) ; \quad Y_{4}=\left(\begin{array}{ccc}
0 & 0 & 0 \\
0 & 0 & 0 \\
1 & 0 & 0
\end{array}\right) .
$$

are still missing. They can be straightforwardly recovered as

$$
X_{4}=\left[X_{1}, Y_{1}\right] \equiv X_{1} Y_{1} ; \quad Y_{4}=\left[Y_{2}, X_{2}\right] \equiv Y_{2} X_{2} .
$$

Thus, by using the set of two triplets $\left(\left(X_{1}, X_{2}, X_{3}\right),\left(Y_{1}, Y_{2}, Y_{3}\right)\right)$ (and their commutation relations) we generate the whole group $S L(3, R)$. In other words, the sequential matrix multiplication of type (26)-(27) generates the group $S L(3, R)$. Qualitatively, this can be seen by writing the square of the metric element of two copies of Lobachevsky planes, $d s_{1,2}^{2}$, in the upper half-plane representation in subspaces $(x, y)$ and $(y, z)$ of the three-dimensional half-space $(x, y>0, z)$ (Siegel space):

$$
\left\{\begin{array}{l}
d s_{1}^{2}=\frac{d x^{2}+d y^{2}}{y^{2}} \text { for } H_{2}^{+}=S L(2, R)_{+} / S O(2)_{+} \\
d s_{2}^{2}=\frac{d y^{2}+d z^{2}}{y^{2}} \text { for } H_{2}^{-}=S L(2, R)_{-} / S O(2)_{-}
\end{array}\right.
$$

The square of the metric element, $d s^{2}=d s_{+}^{2}+d s_{-}^{2}$ in the Siegel space $(x, y>0, z)$ thus reads

$$
d s^{2}=\frac{d x^{2}+2 d y^{2}+d z^{2}}{y^{2}} .
$$

By rescaling $y \rightarrow y / \sqrt{2}$ we arrive at the standard Riemann metric of the Lobachevsky space $H_{3}=S L(3, R) / S O(3)$,

$$
d \tilde{s}^{2}=\frac{1}{2} d s^{2}=\frac{d x^{2}+d y^{2}+d z^{2}}{y^{2}} .
$$

Equation (34) allows us to see that the subspaces $(x, y)$ and $(y, z)$ are two copies of Lobachevsky planes: $\mathrm{H}_{2}^{+}$and $\mathrm{H}_{2}^{-}$. 
One expects that after a sufficient number of depositions, the Haar measure is smeared over the whole group $S L(3, R)$ such that we arrive at the random walk in the symmetric space $H_{3}=S L(3, R) / S O(3)$. The diffusion equation associated with (26) -(27) is governed by the Beltrami-Laplace operator $\Delta_{\mathrm{BL}}$. The most straightforward way of constructing it consists in writing the Casimir $C_{2}$ for infinitesimal transformations on $S L(3, R) / S O(3)$ (see, for example, [38]). Again using the Gauss decomposition, we can decompose the generic matrix $U \in S L(3, R)$ as follows:

$$
U \equiv\left(\begin{array}{lll}
a_{11} & a_{12} & a_{13} \\
a_{21} & a_{22} & a_{23} \\
a_{31} & a_{32} & a_{33}
\end{array}\right)=\left(\begin{array}{ccc}
1 & 0 & 0 \\
y_{1} & 1 & 0 \\
y_{2} & y_{3} & 1
\end{array}\right)\left(\begin{array}{ccc}
e^{\mu_{1}} & 0 & 0 \\
0 & e^{\mu_{2}-\mu_{1}} & 0 \\
0 & 0 & e^{-\mu_{2}}
\end{array}\right)\left(\begin{array}{ccc}
1 & x_{1} & x_{2} \\
0 & 1 & x_{3} \\
0 & 0 & 1
\end{array}\right) .
$$

The infinitesimal generators of the group $S L(3, R)$ in the right representation form are as follows

$$
\begin{aligned}
X_{1}= & \partial_{x_{1}} \\
X_{2}= & e^{\mu_{2}-2 \mu_{1}}\left(\partial_{y_{1}}+y_{3} \partial_{y_{2}}\right)+x_{1} \partial_{\mu_{1}}-x_{1}^{2} \partial_{x_{1}}-x_{1} x_{2} \partial_{x_{2}}-\left(x_{2}-x_{1} x_{3}\right) \partial_{x_{3}} \\
X_{3}= & -2 x_{1} \partial_{x_{1}}+x_{3} \partial_{x_{3}}-x_{2} \partial_{x_{2}}+\partial_{\mu_{1}} \\
X_{4}= & \partial_{x_{2}} \\
Y_{1}= & \partial_{x_{3}}+x_{1} \partial_{x_{2}} \\
Y_{2}= & e^{\mu_{1}-2 \mu_{2}} \partial_{y_{3}}+x_{3} \partial_{\mu_{2}}+x_{2} \partial_{x_{1}}-x_{3}^{2} \partial_{x_{3}} \\
Y_{3}= & x_{1} \partial_{x_{1}}-2 x_{3} \partial_{x_{3}}-x_{2} \partial_{x_{2}}+\partial_{\mu_{2}} \\
Y_{4}= & e^{\mu_{2}-2 \mu_{1}} x_{3} \partial_{y_{1}}-e^{\mu_{1}-2 \mu_{2}} x_{1} \partial_{y_{3}}+\left(e^{-\mu_{1}-\mu_{2}}+y_{3} x_{3} e^{\mu_{2}-2 \mu_{1}}\right) \partial_{y_{2}}+x_{2} \partial_{\mu_{1}} \\
& -\left(x_{1} x_{3}-x_{2}\right) \partial_{\mu_{2}}-x_{1} x_{2} \partial_{x_{1}}+x_{3}\left(x_{1} x_{3}-x_{2}\right) \partial_{x_{3}}-x_{2}^{2} \partial_{x_{2}}
\end{aligned}
$$

The Casimir $C_{2}$ on $S L(3, R)$ has the following form:

$$
C_{2}=X_{1} X_{2}+X_{2} X_{1}+Y_{1} Y_{2}+Y_{2} Y_{1}+X_{4} Y_{4}+Y_{4} X_{4}+\frac{2}{3}\left(X_{3}^{2}+Y_{3}^{2}+X_{3} Y_{3}\right)
$$

Writing $C_{2}$ explicitly in terms of the generators given in Eq. (36), we get:

$$
\begin{aligned}
& \frac{1}{2} C_{2}=\frac{1}{2} \Delta_{\mathrm{BL}}=\frac{1}{3}\left(\partial_{\mu_{1}}^{2}+\partial_{\mu_{2}}^{2}+\partial_{\mu_{1}} \partial_{\mu_{2}}\right)+\partial_{\mu_{1}}+\partial_{\mu_{2}} \\
& \quad+e^{\mu_{1}-2 \mu_{2}} \partial_{x_{3}} \partial_{y_{3}}+e^{-\mu_{1}-\mu_{2}} \partial_{x_{2}} \partial_{y_{2}}+e^{-2 \mu_{1}+\mu_{2}}\left(\partial_{x_{1}} \partial_{y_{1}}+x_{3} \partial_{x_{2}} \partial_{y_{1}}+y_{3} \partial_{x_{1}} \partial_{y_{2}}+x_{3} y_{3} \partial_{x_{2}} \partial_{y_{2}}\right)
\end{aligned}
$$

Let us introduce the "radial" function $Z\left(\mu_{1}, \mu_{2}, t\right)$ such that $W\left(\mu_{1}, \mu_{2}, t\right)=e^{-D t-\left(\mu_{1}+\mu_{2}\right)}$ $\times Z\left(\mu_{1}, \mu_{2}, t\right)$ and apply to (38) the reduction scheme [38] as in (24) fixing $\partial_{x_{2}}=\partial_{y_{2}}=0$. Denoting $\partial_{x_{1}} \partial_{y_{1}}=\varkappa_{1}^{2}$ and $\partial_{x_{3}} \partial_{y_{3}}=\varkappa_{2}^{2}$, where the constants $\varkappa_{1}$ and $\varkappa_{2}$ do not depend on the radial coordinates $\mu_{1}$ and $\mu_{2}$, we get the diffusion equation for the radial part $Z$ :

$$
\partial_{t} Z\left(\mu_{1}, \mu_{2}, t\right)=\frac{D}{3}\left[\partial_{\mu_{1}}^{2}+\partial_{\mu_{2}}^{2}+\partial_{\mu_{1}} \partial_{\mu_{2}}+3 \varkappa_{1}^{2} e^{\mu_{1}-2 \mu_{2}}+3 \varkappa_{2}^{2} e^{-2 \mu_{1}+\mu_{2}}\right] Z\left(\mu_{1}, \mu_{2}, t\right) .
$$

The symmetry of the problem allows us to set in the equation (39) $\varkappa_{1}=\varkappa_{2} \equiv \varkappa$. The corresponding invariant Riemann metric $d s^{2}$ is

$$
\begin{aligned}
\frac{1}{2} d s^{2} & =\frac{1}{2} \operatorname{Tr}\left[g^{-1} d g g^{-1} d g\right] \\
& =\partial_{\mu_{1}}^{2}-\partial_{\mu_{1}} \partial_{\mu_{2}}+\partial_{\mu_{2}}^{2}+e^{2 \mu_{1}-\mu_{2}} \partial_{x_{1}} \partial_{y_{1}}+e^{-\mu_{1}+2 \mu_{2}} \partial_{x_{3}} \partial_{y_{3}}+x_{3} y_{3} e^{\mu_{1}+\mu_{2}} \partial_{x_{1}} \partial_{y_{1}}
\end{aligned} .
$$


As we shall see in the next Section, the diffusion equation (39) coincides with the diffusion equation constructed with the one-dimensional Toda Hamiltonian for two particles in the Chevalley basis [41] with eliminated motion of the center of mass of the system. Note that the fact that we obtain the Casimir $C_{2}$ in (38) in the center-of-mass frame is the consequence of working with $S L(2, R)$ matrices with determinant 1.

\section{Sequential deposition in $N$ columns and one-dimensional Toda chain}

The generic case $N \geq 3$ can be constructed by considering the algebra of $N$ shifted copies of local Lie algebras $s l_{2}$. The Markov chain on the uniform distribution of $N$ elementary $s l_{2}-$ blocks results in growth of the random band-like matrix schematically depicted as follows:

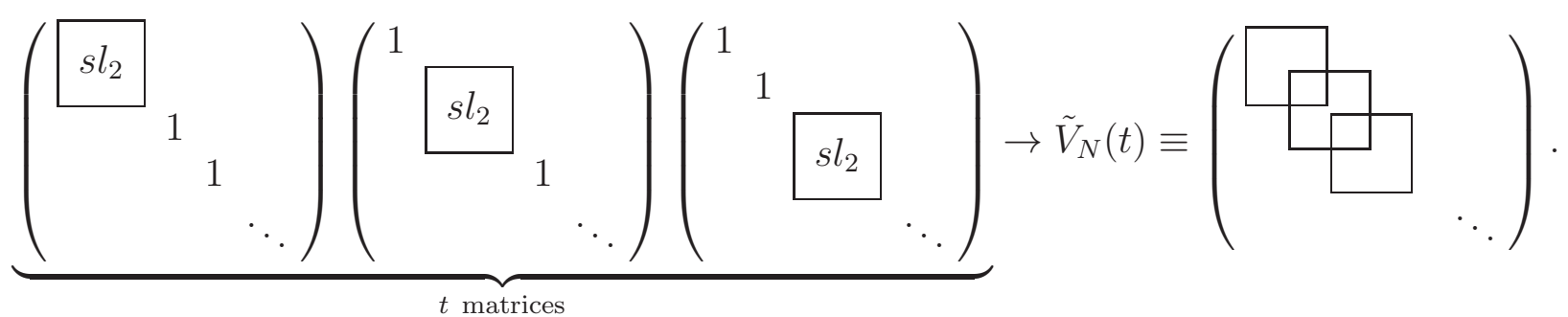

In order to capture the physics of the ballistic growth process it is instructive to begin with non-rigorous, essentially intuitive, construction. Suppose for a moment that $N$ is odd and the deposition events are such that the positions of $s l_{2}$-blocks either coincide, or do not overlap at all. One particular example is shown below:

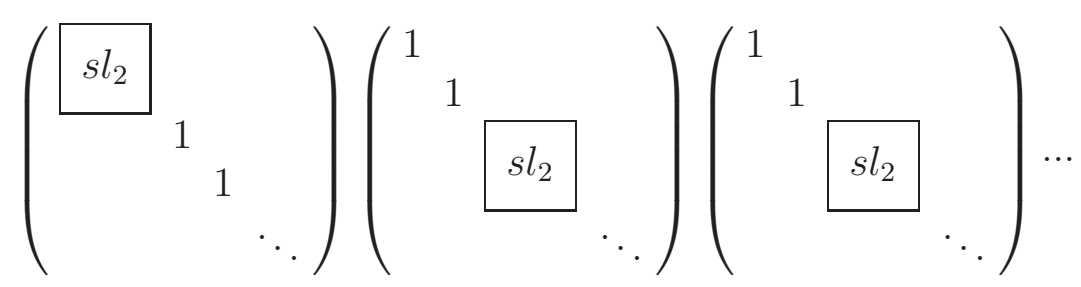

In this case we can easily associate the growth process with the radial diffusion on $N / 2$ noninteracting copies of Lobachevsky plane $H_{2}=S L(2, R) / S O(2)$. By (24) we can easily construct the radial part of the Hamiltonian of $N$ noninteracting particles

$$
H^{(0)}=\sum_{j=1}^{N}\left(p_{j}^{2}+\kappa e^{\mu_{j}}\right),
$$

where $p_{j}=\frac{\partial}{\partial \mu_{j}}$ is the momentum of the particle $j$ (recall that in the deposition process $j$ is the column number), while $\mu_{j}$ is associated with the height in the column $j$.

The simplest way to incorporate the interactions between nearest-neighboring columns which flatten the growing surface, making it less rough, is to replace the Hamiltonian $H^{(0)}$ of noninteracting particles by the following one

$$
H=\sum_{j=1}^{N}\left(p_{j}^{2}+\kappa e^{\mu_{j}-\mu_{j+1}}\right) ; \quad \mu_{N+1} \equiv 0
$$


We obtain this Hamiltonian by direct computations for $N=2,3$ and bring also in Section IV group theoretic arguments in favor of (44) for arbitrary $N$.

First of all, let us demonstrate that the diffusion equation (39) obtained by Gauss decomposition of the group $S L(3, R)$ do coincide with the two-particle Toda Hamiltonian

$$
H(2)=p_{1}^{2}+p_{2}^{2}+\kappa e^{\mu_{1}-\mu_{2}}
$$

under the special choice of the coordinates. Actually, in the frame of the center-of-mass for the $N$-body system, the variables $\mu_{j}(j=1, \ldots, N)$ are transformed as follows [42]

$$
\begin{aligned}
& \mu_{1} \rightarrow \mu_{1}+\frac{\mu_{N+1}}{N+1} ; \quad \mu_{j} \rightarrow-\mu_{j-1}+\mu_{j}+\frac{\mu_{N+1}}{N+1}, 2 \leq j \leq N ; \quad \mu_{N+1} \rightarrow-\mu_{N}+\frac{\mu_{N+1}}{N+1} \\
& p_{j} \rightarrow \frac{1}{N+1}\left[\sum_{i=j}^{N}(N+1-i) p_{i}-\sum_{i=1}^{j-1} i p_{i}\right]+p_{N+1}, 1 \leq j \leq N, \\
& p_{N+1} \rightarrow-\frac{1}{N+1} \sum_{i=1}^{N} i p_{i}+p_{N+1} .
\end{aligned}
$$

The explicit form of the $N$-body Toda Hamiltonian in the coordinates (45) is given in [42]. For our purposes it is sufficient to check the $N=2$-case only. The corresponding result is:

$$
H(2) \rightarrow \frac{1}{3}\left(p_{1}^{2}+p_{2}^{2}+p_{1} p_{2}\right)+\kappa\left(e^{\mu_{1}-2 \mu_{2}}+e^{-2 \mu_{1}+\mu_{2}}\right),
$$

which after the substitution $p_{j}=\frac{\partial}{\partial \mu_{j}}$ gives (for $\varkappa_{1}^{2}=\varkappa_{2}^{2}=\kappa$ ) the diffusion equation (39).

\section{Toda chain and scaling limit of hyperbolic Calogero-type systems}

The Markov chain corresponding to the uniform sequential deposition of elementary $s l_{2}-$ blocks results in a growth of a random band-like matrix $\tilde{V}_{N}(t)$ [as shown schematically in (41)] with a time-dependent bandwidth, $b(t)$, and Gaussian distribution of matrix elements inside the band. Let us repeat that sequential growth means that at each tick of the clock, $t=1,2, \ldots, T$, we multiply the current $N \times N$ matrix $U_{i}^{(t)}$ by an elementary $s l_{2}$-block in a randomly chosen place $i$ along the diagonal [see Eq. (11)] with uniform distribution. The above analysis of "microscopic" transformation of a matrix $\tilde{V}_{N}$ (from $\tilde{V}_{N}(t)$ to $\tilde{V}_{N}(t+1)$ ) results in the diffusion equation for the distribution function $W\left(\mu_{1}, \ldots, \mu_{N}, t\right)$

$$
\frac{\partial}{\partial t} W\left(\mu_{1}, \ldots, \mu_{N}, t\right)=D \sum_{j=1}^{N}\left(\frac{\partial^{2}}{\partial \mu_{j}^{2}}+\kappa e^{\mu_{j}-\mu_{j+1}}\right) W\left(\mu_{1}, \ldots, \mu_{N}, t\right) ; \quad\left(\mu_{N+1}=0\right)
$$

where $\mu_{j}=\ln \lambda_{j}(j=1, \ldots, N)$ and $\lambda_{j}$ are the eigenvalues of a matrix $\tilde{V}_{N}(t)$.

Suppose now that we record changes in matrix $\tilde{V}_{N}$ within some "mesoscopic" time interval, $\Delta t$, thus, producing a sequence $\left\{\tilde{V}_{N}(t=0), \tilde{V}_{N}(\Delta t), \tilde{V}_{N}(2 \Delta t), \ldots, \tilde{V}_{N}(k \Delta t)\right\}(\kappa \gg 1)$. Choosing $\Delta t \sim N$, we ensure the uniform growth of a bandwidth $b$ of a matrix $\tilde{V}_{N}$ with time. Until the width $b$ of the band is of order of unity, one cannot say anything concerning 
the distribution of eigenvalues of $\tilde{V}_{N}(t)$. Meanwhile, it is known (see [43]) that for $b \gg \sqrt{N}$ one can apply the results of the Dorokhov-Mello-Pereyra-Kumar (DMPK) theory [44] to the band matrices. Recall that DMPK theory describes the transmission in a system of $N$ parallel interacting disordered channels. On a formal level this brings us into the subject of probability distribution over time-ordered products of random matrices of size $N \times N$ belonging to some noncommutative noncompact group $G$. This "dynamic" approach deals with the construction of a Fokker-Planck equation for the eigenvalue distribution of random walks on the group $G$ [45, 46]. The DMPK Fokker-Planck equation can be regarded as a heat equation for a radial part of Laplacian acting in the negative curvature symmetric coset space $X^{-}=G / K$, where $K$ is the maximal compact subgroup of $G$ [47, 48, 50].

The standard procedure implies the following steps. Introduce the radial coordinate $x_{i}$ (system of roots) in the Cartan decomposition of $X^{-}$. Note that all $x_{i}(i=1, \ldots, N)$ are ordered: $x_{1}<x_{2}<\ldots<x_{N}$. The distribution of $x_{i}(i=1, \ldots N)$ satisfies the heat equation

$$
\frac{\partial}{\partial t} W(\{\mathbf{x}\}, t)=D \Delta_{\mathrm{BL}}^{(\mathrm{r})} W(\{\mathbf{x}\}, t)
$$

where $D$ is the diffusion coefficient and $\Delta_{\mathrm{BL}}^{(\mathrm{r})}$ is the radial part of Beltrami-Laplace operator in the symmetric space $X^{-}$. The explicit form of $\Delta_{\mathrm{BL}}^{(\mathrm{r})}$ acting in $X^{-}$is well known - see, for example, [48, 49, 51]. Define by $R=\{\alpha\}$ the root space of the Lie algebra $A_{N}$. Let $m_{\alpha}$ be the multiplicity of the root $\alpha$ and consider the case $m_{\alpha}=\beta$ (for short roots) and $m_{\alpha}=1$ (for long roots). We denote by $R^{+}$the subspace of roots in $R$ that is positive with respect to the Weyl chamber of $R$. This allows one to write $\Delta_{\mathrm{BL}}$ as

$$
\Delta_{\mathrm{BL}}^{(\mathrm{r})}=\xi^{-2}\{\mathbf{x}\} \sum_{k=1}^{N} \frac{\partial}{\partial x_{k}} \xi^{2}\{\mathbf{x}\} \frac{\partial}{\partial x_{k}}
$$

where

$$
\xi\{\mathbf{x}\}=\prod_{\alpha \in R_{+}}\left(\sinh x_{\alpha}\right)^{\nu_{\alpha}}=\prod_{i<j}\left|\sinh ^{2} x_{j}-\sinh ^{2} x_{i}\right|^{\beta / 2} \prod_{i}\left|\sinh 2 x_{i}\right|^{1 / 2} .
$$

One sees that the expression (49) for $N=1$ gives the radial part of Beltrami-Laplace operator in the Lobachevsky plane $H_{2}$, where the single root, $\mu_{1}$ of the Cartan algebra is the geodesic distance on a pseudosphere. Recall that for $N=1$ the Liouville-type equation (23) coincides with the random walk in Lobachevsky plane.

The substitution $W\left(\mu_{1}, \ldots, \mu_{N}, t\right)=\xi\left(\mu_{1}, \ldots, \mu_{N}\right) \Psi\left(\mu_{1}, \ldots, \mu_{N}, t\right)$ maps the equations (48), (49) onto the Schrödinger equation

$$
-\frac{\partial}{\partial t} \Psi(\mu, t)=H \Psi(\mu, t),
$$

with $H$ of hyperbolic Calogero-Sutherland-type form

$$
H=-\frac{1}{2} \sum_{j}\left(\frac{\partial^{2}}{\partial x_{j}^{2}}+\frac{1}{\sinh ^{2} 2 x_{j}}\right)+\frac{\beta(\beta-2)}{4} \sum_{j<k}\left(\frac{1}{\sinh ^{2}\left(x_{k}-x_{j}\right)}+\frac{1}{\sinh ^{2}\left(x_{k}+x_{j}\right)}\right)+c
$$

where $c$ is some irrelevant constant. 
It has been pointed out in [51] that the identification of (49)-(52) with the original DMPK equation [45] is possible if and only if the coupling constant in the Calogero-Sutherland Hamiltonian, $g^{2}=\frac{\beta(\beta-2)}{4}$, equals to $\frac{1}{8} m_{\alpha}\left(m_{\alpha}-2\right)|\alpha|^{2}$, where $|\alpha|$ is the root length. While we remind that $m_{\alpha}=\beta$ (for short roots), $m_{\alpha}=1$ (for long roots), in our further consideration we shall not fix specific value of $\beta$, but consider it as an arbitrary parameter. This enables us to establish the connection between hyperbolic Calogero-Sutherland (51)-(52) and Toda (47) in the so-called Inozemtsev limit [52].

To take the Inozemtsev limit in (52), we should rescale the constant $\beta$ simultaneously changing of coordinates $x_{j}(j=1, \ldots, N)$ :

$$
\left\{\begin{array}{l}
\beta=-\sqrt{2 \kappa} e^{\Delta} \\
x_{j}=-\mu_{j}+j \Delta
\end{array} \quad(\Delta \rightarrow \infty) .\right.
$$

Applying (53) to (52) and denoting the limiting Hamiltonian by $\tilde{H}$, we get

$$
-2 \tilde{H}=\frac{1}{2} \sum_{j}\left(\frac{\partial^{2}}{\partial \mu_{j}^{2}}+\kappa e^{2\left(x_{j}-x_{j+1}\right)}\right),
$$

which coincides with the Hamiltonian of Toda chain.

Let us end up this Section by highlight the key observation. i) On one hand, the transmission in multi-channel disordered wires, which corresponds to the Calogero-type system for a special choice of coupling constant, can be described by diffusion in a symmetric space. ii) On the other hand, the diffusion equation in the noncompact space $H_{N}=S L(N, R) / S O(N)$ corresponds to Toda-type system and appears in the consideration of sequential uniform ballistic deposition in multi-column box. The Inozemtsev limit [52] allows to establish the connection between these two different problems i) and ii).

\section{TWO VIEWS ON LAX REPRESENTATION FOR PERIODIC TODA CHAIN}

In this Section we shall briefly consider the NNN ballistic deposition problem with periodic boundary conditions. We have already argued before that the problem is described by the Toda chain system. However we did not pay much attention on the boundary conditions. This is an important issue since the periodic and open Toda chains behave quite differently. It is known that the open chain is related to the motion at $S L(N, R) / S O(N)$ while the periodic chain is related to the motion at the homogenous space of the affine algebra. Moreover the classical motion in the periodic case is described in terms of the Riemann surfaces whose Jacobians are identified with the complex Liouville tori of the dynamical system. The solution to the classical equation in the periodic case is described via the elliptic functions at the Riemann surface while the quantum problem responsible for the NNN ballistic deposition problem is quite involved.

Let us make a couple of general comments concerning the Toda realization of the BD model discussed above with the periodic boundary conditions. First, consider the classical Toda model which allows two different Lax representations. If there are $N$ degrees of freedom 
corresponding to the motion at $S L(N, R) / S O(N)$, the Lax operator can be written either as $2 \times 2$ or $N \times N$ matrix. In the first representation the Lax operator in terms of the conventional phase space coordinates reads as

$$
L_{\text {Toda }}=\left(\begin{array}{cc}
\lambda+p & e^{q} \\
-e^{-q} & 0
\end{array}\right)
$$

This Lax operator acts as the shift operator on the Baker-Akhiezer (BA) function along the sites of the chain. To compare with the discussion above consider the two-component Baker-Akhiezer function $\Psi_{n}=\left(\begin{array}{c}\psi_{n} \\ \chi_{n}\end{array}\right)$. Then the linear problem can be rewritten as

$$
\psi_{n+1}-p_{n} \psi_{n}+e^{q_{n}-q_{n-1}} \psi_{n-1}=\lambda \psi_{n}, \quad \chi_{n}=-e^{q_{n-1}} \psi_{n-1} .
$$

Putting $\chi_{n}=$ const and freezing the dynamical variables in the system we get the equation equivalent to (8). Thus, we come to the following important conclusion:

Statement. The first component of the Baker-Akhiezer function $\Psi_{n}$ plays the role of the finite-temperature partition function of a directed polymer in the random environment. The local heights can be extracted from $\Psi_{n}$ as in (9) by taking logarithm and passing to zerotemperature limit.

This statement approves the link between the NNN ballistic deposition and the "soft" deposition conjectured in the Section \C.

Note that the $2 \times 2$ Lax operator plays the role of the Hamiltonian for the discrete fermionic system while (5) can be identified with the transfer-matrix of Toda model.

The solution to the classical equations of motion is described by the spectral curve

$$
\operatorname{det}[T(\lambda)-\omega]=0 ; \quad T(\lambda)=\prod_{i=1}^{N} L_{i}(\lambda)
$$

where variable $\omega$ corresponds to the quasimomentum for periodic boundary conditions. Generically it is genus $(N-1)$ curve which gets degenerated into the rational one for the non-periodic case. This spectral curve is the Liouville torus for the dynamical system whose degrees of freedom are localized on this curve. In terms of the spectral curve one can define the separated variables that constitute the set of identical noninteracting degrees of freedom. These degrees of freedom are identified with poles of the BA function on the spectral curve.

The question deserving special attention concerns the possibility to find a DouglasKazakov phase transition [53] in a Toda chain for different boundary condition since this transition is responsible for Kardar-Parisi-Zhang scaling (see [54] for details). We plan to discuss this question in a separate publication and as a preliminary remark we outline in Appendix B the connection between solutions of 1D Toda lattice equation and rational solutions of Painlevé II. 
The Toda system can be also written starting from the linear problem

$$
\mathcal{L}=\left(\begin{array}{cccccc}
-p_{1} & e^{\frac{1}{2}\left(q_{2}-q_{1}\right)} & 0 & 0 & \ldots & \frac{1}{w} e^{\frac{1}{2}\left(q_{n}-q_{1}\right)} \\
e^{\frac{1}{2}\left(q_{2}-q_{1}\right)} & -p_{2} & e^{\frac{1}{2}\left(q_{3}-q_{2}\right)} & 0 & \ldots & 0 \\
0 & e^{\frac{1}{2}\left(q_{3}-q_{2}\right)} & -p_{3} & e^{\frac{1}{2}\left(q_{4}-q_{3}\right)} & \ldots & 0 \\
0 & 0 & e^{\frac{1}{2}\left(q_{4}-q_{3}\right)} & -p_{4} & \ldots & 0 \\
\vdots & \vdots & \vdots & \vdots & \ddots & \vdots \\
-w e^{\frac{1}{2}\left(q_{n}-q_{1}\right)} & 0 & 0 & 0 & \ldots & -p_{n}
\end{array}\right),
$$

with the $N$-component Baker-Akhiezer function $\Phi=\left\{e^{-q_{n} / 2} \psi_{n}\right\}$. This leads us to the spectral curve

$$
\operatorname{det}_{n \times n}(\mathcal{L}(w)-\lambda)=0,
$$

which is equivalent to the spectral curve in the $2 \times 2$ representation and reads as

$$
\omega+\omega^{-1}=P_{N}(\lambda) .
$$

The symmetry of two different representations of Lax operator by $2 \times 2$ or $N \times N$ matrices can be interpreted as "time"-like (a) and "space"-like (b) representations schematically depicted in Fig. 5. Geometrically this symmetry means the rotation of the picture in the homogeneous space - see [55] for details. In terms of ballistic deposition the time-like representation can be visualized as growth along the time running since the first deposition event, while the space-like representation can be associated with growth "along a surface" of the heap (i.e. in the direction orthogonal to the time axis).

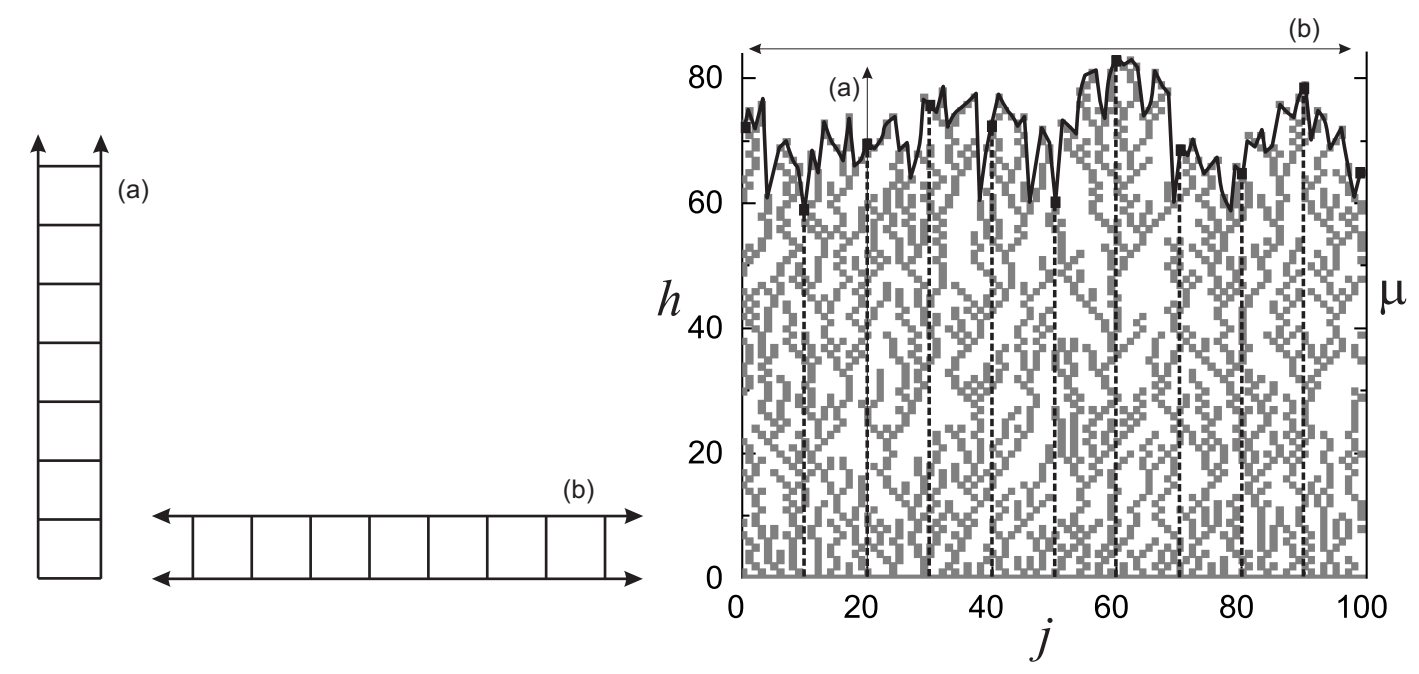

Figure 5. Two possibilities of constructing Lax representation for Toda chain: (a) "time-like" and (b) "space-like". The visualization of these representations in terms of growing heap is shown nearby.

Let us conclude the reminder about two types of Lax representations by noting that ideologically similar description is allowed for the one-dimensional Anderson model. Two 
different points of view on this problem, described below, deal nothing with integrability, however they have exactly the same physical interpretation as two different Lax representations of 1D Toda chain. Following [56], consider the electron hopping on the one-dimensional lattice described by a tight-binding one-dimensional Schrödinger equation with site disorder. Denote by $\psi_{j}$ and $U_{j}$ the wave function and the random potential on the lattice site $j$. Then the eigenvalue problem is given by the equation

$$
\psi_{j-1}+U_{j} \psi_{j}(x)+\psi_{j+1}=E \psi_{j}(x) .
$$

Equation (61) admits two representations. Introducing the two-component vector $\left(\psi_{j+1}, \psi_{j}\right)$, we can rewrite (61) as

$$
\left(\begin{array}{c}
\psi_{j+1} \\
\psi_{j}
\end{array}\right)=L_{j}\left(\begin{array}{c}
\psi_{j} \\
\psi_{j-1}
\end{array}\right) ; \quad L_{j}=\left(\begin{array}{cc}
E-U_{j}-1 \\
1 & 0
\end{array}\right)
$$

which has similarity with (58). From the other hand, Eq. (61) has representation in terms of spectral problem for $N \times N$ tridiagonal matrix $\mathcal{L}$ applied to the vector $\Psi=\left\{\psi_{1}, \psi_{2}, \ldots \psi_{N}\right\}$ :

$$
\mathcal{L}=\left(\begin{array}{cccccc}
E-U_{1} & -1 & 0 & 0 & \ldots & a_{1 n} \\
-1 & E-U_{2} & -1 & 0 & \ldots & 0 \\
0 & -1 & E-U_{3} & -1 & \ldots & 0 \\
0 & 0 & -1 & E-U_{4} & \ldots & 0 \\
\vdots & \vdots & \vdots & \vdots & \ddots & \vdots \\
a_{n 1} & 0 & 0 & 0 & \cdots & E-U_{N}
\end{array}\right)
$$

It might be interesting to check whether such a structural analogy between two Lax representations of Toda chain and two descriptions of Anderson model has more deep physical grounds than just a formal similarity.

\section{CONCLUSION}

In this paper we have reformulated the NNN ballistic deposition process in terms of timeordered product of the generators of a local group $L_{N}$ (see the Definition 1 ). The generators of this group are the $N \times N$-matrices consisting of a single $s l_{2}$-block randomly located along the diagonal. We have related the Markov dynamics on $L_{N}$ with the diffusion in the metric space $H_{N}=S L(N, R) / S O(N)$.

Let us summarize the main results of our work:

- By constructing the Beltrami-Laplace operator on $H_{N}$ in an appropriate system of coordinates obtained via Gauss decomposition, we claim the existence of the integrable structure behind the NNN deposition problem.

- We demonstrate the relation between the NNN growth and dynamics of the 1D Toda chain where the number of sites in the chain coincides with the number of the sites in the deposition problem. The largest geodesic distance in the corresponding symmetric space $H_{N}=S L(N, R) / S O(N)$ is identified with the maximal local heights in the deposition process. 
- Taking the Inozemtsev limit in the hyperbolic Calogero-Sutherland model we uncover the connection between the ballistic deposition process and the transmission in multichannel random wires. This connection certainly deserves further study.

It is worth pointing out the relation between the fluctuations of the partition function of a directed polymer at finite temperatures and the quantum Toda lattice has been obtained by N. O'Connell in a model of a semi-continuous directed polymer model with the length related to increments of Brownian motions [57]. Within this model it was obtained that the free energy, as a function of $t$, behaves like the first component of a process in $\mathbb{R}^{N}$ which also satisfies a diffusion equation with a Toda Hamiltonian. The extension of this work to statistics of vicious random walks with "a long-ranged killing term" has been considered in a recent work [58]. It would be very interesting to establish correspondence between the subject of our work, the problems analyzed in [57, 58] and eventually to the Yang-Mills formulation of the statistics of vicious random walks developed recently in [54].

Let us outline the possible directions for further investigation. It seems to us that the most interesting case corresponds to the periodic boundary conditions. In this case the deposition problem gets mapped onto the periodic Toda chain whose solution to the classical equation of motion is described by the Riemann surface. In this case one could define the prepotential which yields the solution of the dispersionless Whitham hierarchy similar to the one considered in [59]. We plan to discuss the periodic case separately.

Note the analogy between the ballistic deposition and the Laplacian growth (LG). In LG case the integrable system is defined as well, where the natural degrees of freedom are attributed to the shape of the droplet while the Whitham times get identified with the momenta of the droplet. The initial evolution equation selects the particular solution of the Whitham hierarchy. In the BD case the local heights play the role of the Toda degrees of freedom. From the physical point of view the difference between the LG and BD consists only in deposition rules: in Laplacian growth the particles follow Brownian trajectories until they are attached to the cluster, while in ballistic depositions the particles follow straight parallel trajectories as in a rain. The passage from LG to BD consists in "elongation" of Brownian trajectories in an external field. This point of view is the subject of our forthcoming work.

Our final remark concerns the existence of shocks in Toda chain [65]. In [66] we have demonstrated that the discrete stochastic equation describing the BD process in the NNN model can be naturally represented in terms of a dynamic programming language. This dynamic programming point of view allowed for a systematic translation of the study of growing clusters and channels separating them ("crevisses") into the language of maximizers and shocks in discrete equations of Burgers or Hamilton-Jacobi type. Whether these geometrical properties of a growing heap have interpretation in terms of shocks in Toda chain, is an open and challenging question.

\section{Acknowledgments}

The authors are grateful to A. Comtet, Y. Fyodorov, S. Majumdar and V. Priezzhev for encouraging discussions. The work of A.G. was partially supported by grants RFBR-09-0200308 and CRDF-RUP2-2961-MO-09. A.G. is grateful to Simons Center for Geometry and Physics and KITP at UCSB, where part of this work has been done, for the hospitality and support. 


\section{Appendix A: The connection between the columnar heights and coordinates in the symmetric space}

1. We expect for $N \geq 2$ the following linear relation between heights $\left\{h_{1}, \ldots, h_{N}\right\}$ and geodesic distances $\left\{\mu_{1}, \ldots, \mu_{N}\right\}$ to hold:

$$
\max \left\{h_{1}, \ldots, h_{N}\right\}=\gamma_{N} \max \left\{\mu_{1}, \ldots, \mu_{N}\right\}
$$

where $\gamma_{N}$ is some constant, $\mu_{j}=\ln \lambda_{j}(j=1, \ldots, N)$ and $\lambda_{j}$ are the eigenvalues of the matrix $\tilde{V}_{t}$, which is the product of generators of locally free semigroup $F_{N}^{+}$. The behavior (A1) is supported by numerical simulations of NNN uniform ballistic deposition in a $N-$ columnar bounding box, considered as: i) sequential adding of $T$ elementary blocks with NNN interactions, and ii) time-ordered multiplication of $T$ matrices-generators of the locally free semigroup $F_{N}^{+}$.

In the Fig. 6 (a) we plot the ratio $\gamma_{N}(T)=\max \left\{h_{1}, \ldots, h_{N}\right\} / \max \left\{\mu_{1}, \ldots, \mu_{N}\right\}$ in the interval $T=1000 \div 10000$ for fixed values of $N$ (for better visibility the coordinates $\left(\gamma_{N}, 1 / T\right)$ are used). We see that for each $N$ the coefficient $\gamma_{N}(1 / T)$ tends for $T \rightarrow \infty$ to some selfaveraged value $\gamma_{N}(0)$.

The figure Fig. 6 (b) demonstrates the dependence of $\gamma_{N}(0)$ on $N$ for $N=2,4,6,8$, 10, 20, 30, 40. The value $\gamma_{N}(0)$ saturates very rapidly with increasing of $N$ and reaches the limiting value $\approx 0.8$.

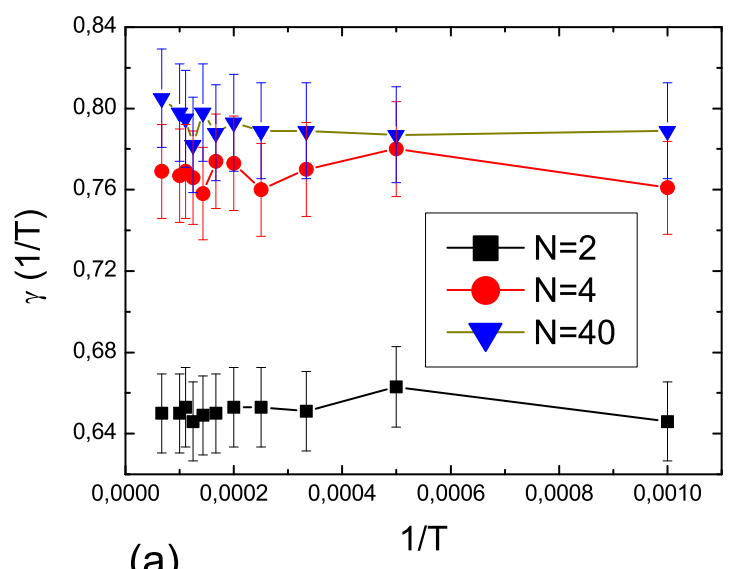

(a)

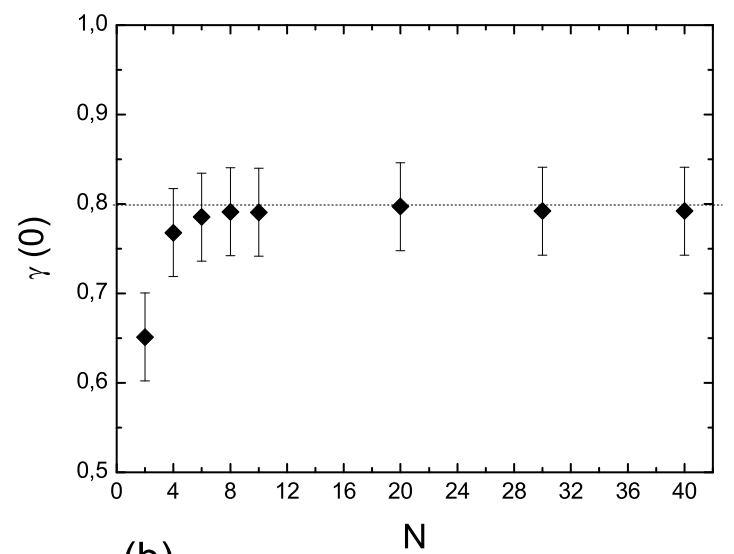

(b)

Figure 6. Linear dependence of maximal height for NNN-deposition on the logarithm of the maximal eigenvalue in corresponding matrix representation: a) dependence of $\gamma_{N}$ on $1 / T$ for fixed $N$; b) dependence of $\gamma_{N}(0)$ on different $N$.

The results of simulation depicted in Fig. 6 demonstrate the linear dependence of the maximal height on the logarithm of the largest eigenvalue in associated matrix representation. Moreover, for $N \gg 1$ the coefficient $\gamma_{N}$ becomes independent on $N$.

2. The relation between the height in $N=2$-ballistic deposition and the corresponding hyperbolic distance in $H_{2}=S L(2, R) / S O(2)$ can be easily established. To see that, note that the height in the column is in one-to-one correspondence with the shortest distance on 
the corresponding Cayley graph - see Fig. 4 of the Section IIC. Let us discuss in details the two-columnar deposition and show that the maximal height in two neighboring columns, i.e. the average number of steps along a Cayley graph is linearly proportional to the hyperbolic distance, $d$, in the space $\mathrm{H}_{2}$. We use some facts about the isometric embedding of graphs in hyperbolic planes and uniform Markov processes in hyperbolic domains - for more details see, for example, [67].

Consider the hyperbolic Poincaré upper half-plane $H_{2}=\{z \in C, \operatorname{Im}(z)>0\}$. The matrix representation of the generators of the group $\Gamma_{2}$ is well known (see, for example [40]), however for our purposes it is more convenient to take a basis consisting of the composition of standard fractional-linear transform and complex conjugacy. Namely, denoting by $\bar{z}$ the complex conjugate of $z$, we consider the following action

$$
\left(\begin{array}{ll}
a & b \\
c & d
\end{array}\right): z \rightarrow \frac{a \bar{z}+b}{c \bar{z}+d}
$$

A possible set of generators is then:

$$
h_{0}=\left(\begin{array}{cc}
1 & -2 / \sqrt{3} \\
0 & -1
\end{array}\right), h_{1}=\left(\begin{array}{cc}
1 & 2 / \sqrt{3} \\
0 & -1
\end{array}\right), h_{2}=\left(\begin{array}{cc}
0 & 1 / \sqrt{3} \\
\sqrt{3} & 0
\end{array}\right)
$$

Choosing the point $\left(x_{0}, i y_{0}\right)=(0, i)$ as the tree root - see fig, 7 , any vertex on the tree is associated with an element $V_{n}=\prod_{k=1}^{n} h_{\alpha_{k}}$ where $\alpha_{k} \in\{0,1,2\}$ and is parameterized by its complex coordinates $z_{n}=V_{n}\left((-1)^{n} i\right)$ in the hyperbolic plane.

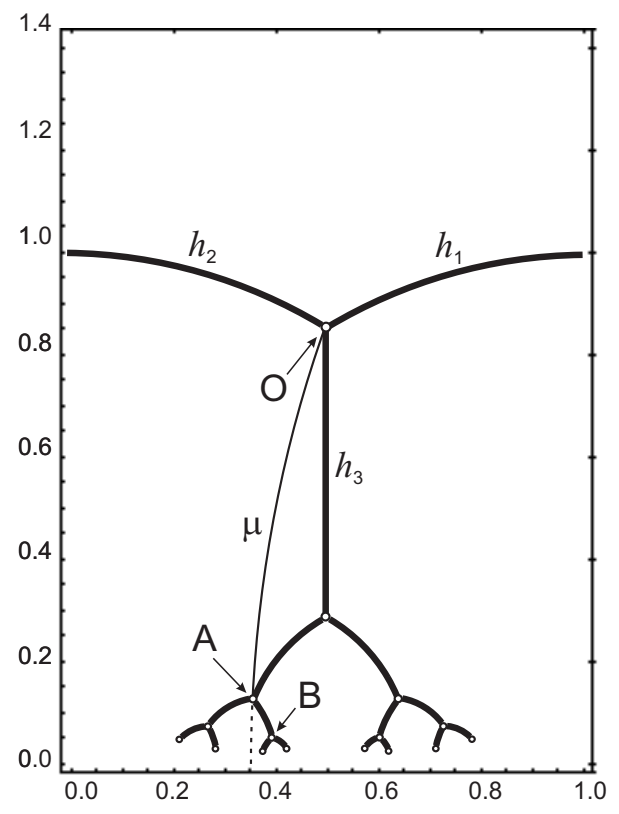

Figure 7. Poincaré hyperbolic upper half-plane $H_{2} . \quad A$ and $B$ are two neighboring vertices of Cayley tree isometrically embedded into $\mathrm{H}_{2} ; d$ is the hyperbolic distance between points $A$ and $B$.

For $H_{2}=S L(2, R) / S O(2)$ one can identify an element with its class of equivalence of $S O(2)$. If one denotes by $\mu\left(V_{n}\right) \equiv \mu\left(i, z_{n}\right)$ the hyperbolic distance between $i$ and $z_{n}$, the following identity holds

$$
2 \cosh \mu\left(V_{n}\right)=\operatorname{Tr}\left(V_{n} V_{n}^{\dagger}\right)
$$


where dagger denotes transposition.

Let $W_{n}(\mu)$ be the probability to find the tree vertex in the generation $n$ at the hyperbolic distance $\mu$ from the root point. In terms of deposition process, $n$ is the average height of two neighboring columns). It means that we are looking for the distribution of the traces for matrices $V_{n}$ which are irreducible products of $n$ sequential multiplications. If we denote by $\mu\left(V_{n}\right)$ the irreducible length of the word represented by the matrix $V_{n}$, then $V_{n}$ is irreducible if and only if $\mu\left(V_{n}\right)=n$. Such a word enumeration problem is simple in case of the group $\Gamma_{2}$, because of its free product structure: $\Gamma \sim Z_{2} \otimes Z_{2} \otimes Z_{2}$. Indeed, if $V_{n}=\prod_{k=1}^{n} h_{\alpha_{k}}$ one has $\mu\left(V_{n}\right)=n$ if and only if $h_{\alpha_{k}} \neq h_{\alpha_{k-1}} \forall k$. Hence we have to study the behavior of the random matrix $V_{n}$, generated by the following Markovian process

$$
V_{n+1}=V_{n} h_{\alpha_{n+1}} \text { with } \alpha_{n+1}= \begin{cases}\left(\alpha_{n}+1\right) \bmod 3 & \text { with probability } \frac{1}{2} \\ \left(\alpha_{n}+2\right) \bmod 3 & \text { with probability } \frac{1}{2}\end{cases}
$$

One can use the standard methods of random matrices and consider the entries of the $2 \times 2$-matrix $V_{n}$ as a 4 -vector $\mathcal{V}_{n}$. The transformation $V_{n+1}=V_{n} h_{\alpha}$ reads

$$
\mathcal{V}_{n+1}=\left(\begin{array}{cc}
h_{\alpha}^{\dagger} & 0 \\
0 & h_{\alpha}^{\dagger}
\end{array}\right) \mathcal{V}_{n}
$$

This block-diagonal form allows one to restrict ourselves to the study of one of two 2vectors, composing $\mathcal{V}_{n}$, say $v_{n}$. Parameterizing $v_{n}=\left(\varrho_{n} \cos \theta_{n}, \varrho_{n} \sin \theta_{n}\right)$ and using the relation $\mu\left(V_{n}\right) \equiv \mu_{n} \simeq 2 \ln \varrho_{n}$ valid for $n \gg 1$, one gets a recursion relation $v_{n+1}=h_{\alpha}^{\dagger} v_{n}$ in terms of hyperbolic distance $\mu_{n}$ :

$$
\mu_{n+1}=\mu_{n}+\ln \left[\frac{5}{3}+\frac{4}{3} \cos \left(2 \theta_{n}+\varphi_{\alpha}\right)\right]
$$

where $\varphi_{\alpha}$ depends on the transform $h_{\alpha}$ through $\varphi_{\alpha}=(2 \alpha-1) \pi / 3(\alpha=0,1,2)$, while for the angles one gets straightforwardly

$$
\tan \theta_{n+1}=h_{\tilde{\alpha}}\left(\tan \theta_{n}\right)
$$

and the change $\alpha \rightarrow \tilde{\alpha}$ means the substitution $(0,1,2) \rightarrow(1,0,2)$. Action of $h_{\alpha}$ is still fractional-linear. The properties of the invariant measure $\nu_{\alpha}(\theta)$ have been discussed by Gutzwiller and Mandelbrot [68]. We are now led to compute the Lyapunov exponent, $\gamma$ which is

$$
\gamma=\lim _{n \rightarrow \infty} \frac{\langle\mu\rangle}{n}=\int_{0}^{\frac{\pi}{3}} d \theta \mu_{1}\left(\theta-\frac{\pi}{6}\right) \ln \left(\frac{5}{3}+\frac{4}{3} \cos 2 \theta\right)
$$

The numerical value of $\gamma$ can be obtained by means of semi-numerical procedure which involves the numerical information about the invariant measure $\mu_{1}(\theta)$ [67, 68]. One finally gets: $\gamma \approx 0.79$. By this we prove the linear relation $\mu=\gamma n$ between hyperbolic distance, $\mu$, in $H_{2}$ and average height in two neighboring columns, $n$. 


\section{Appendix B: Minimal action principle for Toda system and rational solutions of Painlevé II}

Represent the partition function $W$ in the form of Feynmann-Kac path integral with the Lagrangian obtained by Legendre transformation from Toda Hamiltonian (44):

$$
W\left(\mu_{1}, \ldots, \mu_{N}, t\right)=\int \ldots \int \mathcal{D}\{\mu\} \exp \left\{-\int_{0}^{t} d s\left[\sum_{j=1}^{N} \dot{\mu}_{j}^{2}(s)+V\left(\mu_{j}(s), \mu_{j+1}(s)\right)\right]\right\},
$$

where $V\left(\mu_{j}, \mu_{j+1}\right)=e^{\mu_{j}-\mu_{j+1}}$ is the Toda potential. Minimizing the corresponding action, we arrive at the standard Euler equations, which describe the dynamics of 1D Toda chain:

$$
\ddot{\mu}_{j}(s)=e^{\mu_{j}-\mu_{j+1}}-e^{\mu_{j+1}-\mu_{j+2}},
$$

where $\ddot{\mu}_{j}(s) \equiv \frac{d^{2}}{d s^{2}} \mu_{j}(s)$. There are many equivalent representations of Toda chain equation (B2). Among the most known, is the bilinear form of (B22) (see, for example, [60]):

$$
\tau_{j}^{\prime \prime} \tau_{j}-\left(\tau_{j}^{\prime}\right)^{2}=\tau_{j+1} \tau_{j-1}
$$

where $\tau_{j}^{\prime}=\frac{d}{d s} \tau_{j}, \tau_{j}^{\prime \prime}=\frac{d^{2}}{d s^{2}} \tau_{j}$ and

$$
\mu_{j}=\ln \frac{\tau_{j-1}}{\tau_{j}} .
$$

The tau-function $\tau_{n}$ can be written in the determinant form (see, for example, [61] )

$$
\tau_{j}=\operatorname{det}\left(\begin{array}{cccc}
\varphi & \frac{d}{d s} \varphi & \cdots & \left(\frac{d}{d s}\right)^{j-1} \varphi \\
\frac{d}{d s} \varphi & \left(\frac{d}{d s}\right)^{2} \varphi & \cdots & \left(\frac{d}{d s}\right)^{j} \varphi \\
\vdots & \vdots & \ddots & \vdots \\
\left(\frac{d}{d s}\right)^{j-1} \varphi & \left(\frac{d}{d s}\right)^{j} \varphi & \cdots & \left(\frac{d}{d s}\right)^{2 j-2} \varphi
\end{array}\right) .
$$

The function $\varphi$ for open boundary conditions is an arbitrary function of $s$. Applying a gauge transformation to $\tau_{j}$

$$
\frac{d^{2}}{d s^{2}} \ln \tau_{j}(s)=\frac{d^{2}}{d s^{2}} \ln \sigma_{j}(s)+p q,
$$

with arbitrary $p$ and $q$, one arrives at the equation

$$
\sigma_{j}^{\prime \prime} \sigma_{j}-\left(\sigma_{j}^{\prime}\right)^{2}=\sigma_{j+1} \sigma_{j-1}-p q \sigma_{j}^{2},
$$

where $\sigma_{-1}=p, \sigma_{0}=1, \sigma_{1}=q$. Equation (B7) resembles the differential-difference equation that defines Yablonskii-Vorob'ev polynomials [62], $Q_{n}(z)$ :

$$
Q_{j}^{\prime \prime}(z) Q_{j}(z)-\left(Q_{j}^{\prime}(z)\right)^{2}=-\frac{1}{4} Q_{j+1} Q_{j-1}+\frac{z}{4} Q_{j}^{2}(z),
$$

with $Q_{0}=1, Q_{1}=z$ and the derivatives taken with respect to $z$. To establish the precise connection between ( $(\mathrm{B} 7)$ and $(\overline{\mathrm{B} 8})$, let us make the shift in (B7): $\sigma_{j} \rightarrow A^{j^{2}} \sigma_{j}$ and then fix the values of $A, p$, and $q$ to satisfy conditions: $A=\frac{i}{2}, p q=-\frac{z}{4}$. 
It is known [63, 64] that the Yablonskii-Vorob'ev polynomials $Q_{n}(z)$ are connected to the rational solutions of Painlevé II. Namely, the combination

$$
w_{j}(z)=\frac{d}{d z} \ln \frac{Q_{j-1}(z)}{Q_{j}(z)} \equiv \frac{Q_{j-1}^{\prime}(z)}{Q_{j-1}(z)}-\frac{Q_{j}^{\prime}(z)}{Q_{j}(z)}
$$

gives the rational solutions of the Painlevé II equation

$$
w^{\prime \prime}(z)=2 w^{3}(z)+z w(z)+\alpha \quad(\alpha=j \in \mathbb{Z}) .
$$

So, the classical optimal trajectory solution for Toda Hamiltonian can be linked by equations (B1) $-($ B10 $)$ to the rational solutions of the Painlevé II equation.

[1] M.A. Herman and H. Sitter, Molecular Beam Epitaxy: Fundamentals and Current, (Springer: Berlin, 1996).

[2] P. Meakin, Fractals, Scaling, and Growth Far From Equilibrium, (Cambridge University Press: Cambridge, 1998).

[3] M. Prähofer and H. Spohn, J. Stat. Phys. 1081071 (2002).

[4] J. Baik and E.M. Rains, J. Stat. Phys. 100523 (2000).

[5] K. Johansson, Comm. Math. Phys. 242277 (2003).

[6] B.B. Mandelbrot, The Fractal Geometry of Nature, (Freeman: New York, 1982).

[7] P. Meakin, P. Ramanlal, L. M. Sander, and R. C. Ball, Phys. Rev. A 345091 (1986).

[8] J. Krug and P. Meakin, Phys. Rev. A 402064 (1989).

[9] D. Blomker, S. Maier-Paape, and T. Wanner, Interfaces and Free Boundaries 3465 (2001).

[10] Z. Toroczkai, G. Korniss and S. Das Sarma, R. K. P. Zia, Phys. Rev. E 62276 (2000).

[11] S.N. Majumdar and S. Nechaev, Phys. Rev. E 69011103 (2004).

[12] M. Kardar, G. Parisi and Y.-C. Zhang, Phys. Rev. Lett. 56889 (1986); T. Halpin-Healy and Y.-C. Zhang, Phys. Rep. 254215 (1995).

[13] G. Costanza, Phys. Rev. E 556501 (1997).

[14] F.D.A. Aarao Reis, Phys. Rev. E 63056116 (2001).

[15] E. Katzav and M. Schwartz, Phys. Rev. E 70061608 (2004).

[16] K. Johansson, Comm. Math. Phys. 209437 (2000).

[17] M. Prähofer and H. Spohn, Phys. Rev. Lett. 844882 (2000); M. Prähofer and H. Spohn, J. Stat. Phys., 108, 1071 (2002).

[18] C.A. Tracy and H. Widom, Commun. Math. Phys. 159151 (1994).

[19] L. Miettinen, M. Myllys, J. Merikoski, J. Timonen, Eur. Phys. J. B 46, 55 (2005).

[20] K. A. Takeuchi, M. Sano, Phys. Rev. Lett. 104, 230601 (2010).

[21] K. A. Takeuchi, M. Sano, T. Sasamoto, H. Spohn, Sci. Rep. (Nature) 1, 34 (2011).

[22] J. G. Amar and F. Family, Phys. Rev. E 471595 (1993).

[23] B.H. Gildinga, M. Gueddab and R. Kersner, J. Math. Analysis App. 284733 (2003).

[24] J. Bec and K. Khanin, Phys. Rep., 4471 (2007).

[25] R.E. Bellman, Dynamic Programming (Dover Publications: 2003).

[26] T. Nagatani, Phys. Rev. E 58700 (1998); N. Nishinari and D. Takahashi, J. Phys. A 315439 (1989). 
[27] S.N. Majumdar, Random Matrices, the Ulam Problem, Directed Polymers \& Growth Models, and Sequence Matching, Les Houches Lecture Notes, Summer school "Complex Systems" (Les Houches, 2006).

[28] A.M. Vershik, In: Topics in Algebra 26 (pt.2) 467 (1990) (Banach Center Publication, Warszawa); A.M. Vershik, Proc. Am. Math. Soc. 1481 (1991).

[29] A.M. Vershik, S. Nechaev and R. Bikbov, Comm. Math. Phys. 212469 (2000).

[30] X.G. Viennot, Ann. N.Y. Ac. Sci. 576542 (1989).

[31] P. Cartier and D. Foata, Lect. Not. Math. 85 (New York-Berlin: Springer, 1969).

[32] E.S. Esyp, I.V. Kazachkov and V.N. Remeslennikov, Groups, Languages, Algorithms Contemp. Math. 378319 (2005), arXiv:math/0512401

[33] C. Series, J. London Math. Soc. (2) 3169 (1985).

[34] M.E. Gertsenshtein and V.B. Vasil'ev, Theory Prob. Appl., 4391 (1959).

[35] F.I. Karpelevich, V.N. Tutubalin and M.G. Shur, Theory Prob. Appl., 4399 (1959).

[36] S. Helgason, Differential Geometry, Lie Groups and Symmetric Spaces, (Academic Press: New-York, 1985)

[37] H. Fürstenberg, Trans. Amer. Math. Soc. 198377 (1963).

[38] A. Gerasimov, S. Kharchev, A. Morozov, M. Olshanetsky, A. Marshakov, A. Mironov, Int. J. Mod. Phys. A, 122523 (1997).

[39] A. Comtet and C. Monthus, J. Phys. A: Math. Gen. 291331 (1996)

[40] A. Terras, Harmonic Analysis on Symmetric Spaces and Applications I, (Springer: New-York, 1985).

[41] R.W. Cartier, Simple Groups of Lie Type, (New York: John Wiley \& Sons, 1972)

[42] A. Anderson, J. Math. Phys. 371349 (1996)

[43] Y.V. Fyodorov and A.D. Mirlin, Int. J. Mod.Phys. B 83795 (1994)

[44] O. N. Dorokhov, JETP Lett. 36, 318 (1982); P.A. Mello, P. Pereyra and N. Kumar, Ann. Phys. 181, 290 (1988).

[45] C.W.J. Beenakker and B. Rajaei, Phys. Rev. B 497499 (1994)

[46] J.-L. Pichard, In: Waves and Imaging through Complex Media (ed.: P. Sebbah, Kluwer Academic Publishers), 125 (2001) arXiv:cond-mat/0107437

[47] A. Hüffmann, J. Phys. A 235733 (1990).

[48] M.A. Olshanetsky and A.M. Perelomov, Physics Reports, 94313 (1983)

[49] M.A. Olshanetsky and A.M. Perelomov, Funct. Anal. Appl. 12121 (1978) arXiv:math-ph/0203031

[50] M. Caselle and U. Magnea, Physisc Reports 39441 (2004).

[51] M. Caselle, Phys. Rev. Lett. 74, 2776 (1995)

[52] Inozemtsev, Comm. Math. Phys., 121629 (1989).

[53] M. R. Douglas and V. A. Kazakov, Phys. Lett. B 319219 (1993).

[54] P.J. Forrester, S. N. Majumdar and G. Schehr, Nucl. Phys. B 844500 (2011) arXiv:1009.2362.

[55] A. Gorsky, S. Gukov and A. Mironov, Nucl. Phys. B 517409 (1998)

[56] F. Haake, Quantum Signatures of Chaos, (Springer: Berlin, 2001)

[57] N. O'Connel, arXiv:0910.0069.

[58] M. Katori, arXiv:1110.1845.

[59] A. Gorsky, A. Marshakov, A. Mironov and A.Morozov, Nucl. Phys. B 527690 (1998) arXiv:hep-th/9802007.

[60] R. Conte, F. Magri, M. Musette, J. Satsuma and P.Winternitz, Direct and Inverse Methods in Nonlinear Evolution Equations, (Springer: Berlin, 2003) 
[61] K. Kajiwara, T. Masuda, M. Noumi, Y. Ohta, Y. Yamada, arXiv:solv-int/9908007; K. Iwasaki, K. Kajiwara, T. Nakamura, arXiv:nlin/0112043 N. Joshi, K. Kajiwara, M. Mazzocco, arXiv:nlin/0406035;

[62] A.I. Yablonskii, On rational solutions of the second Painlevé equation, Vesti AN BSSR, Ser. Fiz.-Tech. Nauk, no. 3, 30 (1959) (in Russian); A.P. Vorob'ev, On the rational solutions of the second Painlevé equations, Differ. Uravn. 179 (1965) (in Russian); P.A. Clarkson, Séminaires \& Congrès 14, 21 (2006).

[63] K. Kajiwaraa, K. Yamamotoa, Y. Ohta, Phys. Lett. A 232189 (1997).

[64] P. Roffelsen, arXiv:1012.2933.

[65] S. Kamvissis, Physica D 65, 242 (1993); P. Deift, S. Kamvissis, T. Kriecherbauer and X. Zhou, Comm. Pure and Applied Math. 493543 (1996).

[66] K. Khanin, S. Nechaev, G. Oshanin, A. Sobolevski and O. Vasilyev, Phys. Rev. E 82061107 (2010) arXiv:1006.4576].

[67] A. Comtet, S. Nechaev and R. Voituriez, J. Stat. Phys. 102203 (2001).

[68] M. Gutzwiller and B. Mandelbrot, Phys. Rev. Lett. 60673 (1988) 